University of Nebraska - Lincoln

DigitalCommons@University of Nebraska - Lincoln

3-7-2002

\title{
Thermodynamics and bioenergetics
}

Yasar Demirel

Virginia Polytechnic Institute and State University, ydemirel2@unl.edu

S.I. Sandler

Follow this and additional works at: https://digitalcommons.unl.edu/chemengthermalmech

Part of the Heat Transfer, Combustion Commons

Demirel, Yasar and Sandler, S.I., "Thermodynamics and bioenergetics" (2002). Papers in Thermal Mechanics. 7.

https://digitalcommons.unl.edu/chemengthermalmech/7

This Article is brought to you for free and open access by the Chemical and Biomolecular Engineering Research and Publications at DigitalCommons@University of Nebraska - Lincoln. It has been accepted for inclusion in Papers in Thermal Mechanics by an authorized administrator of DigitalCommons@University of Nebraska - Lincoln. 

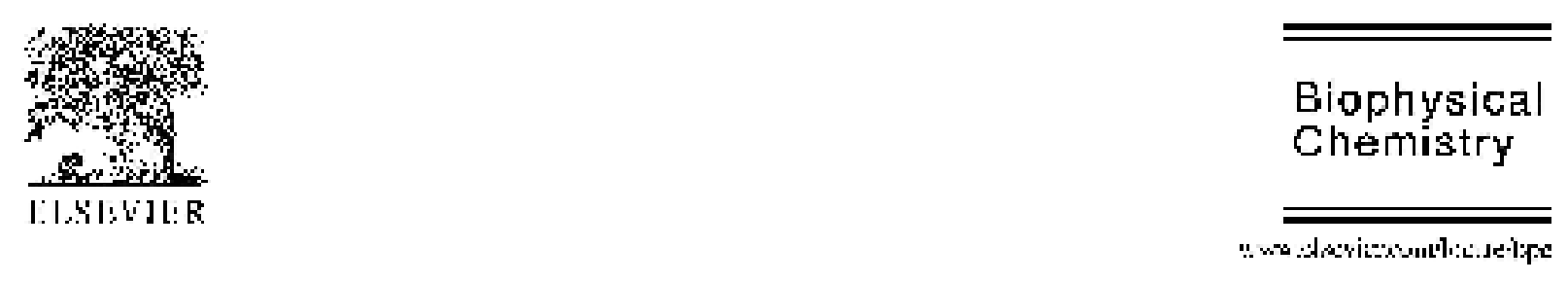

\title{
Thermodynamics and bioenergetics
}

\begin{abstract}
Bioenergetics is concerned with the energy conservation and conversion processes in a living cell, particularly in the inner membrane of the mitochondrion. This review summarizes the role of thermodynamics in understanding the coupling between the chemical reactions and the transport of substances in bioenergetics. Thermodynamics has the advantages of identifying possible pathways, providing a measure of the efficiency of energy conversion, and of the coupling between various processes without requiring a detailed knowledge of the underlying mechanisms. In the last five decades, various new approaches in thermodynamics, nonequilibrium thermodynamics and network thermodynamics have been developed to understand the transport and rate processes in physical and biological systems. For systems not far from equilibrium the theory of linear non-equilibrium thermodynamics is used, while extended nonequilibrium thermodynamics is used for systems far away from equilibrium. All these approaches are based on the irreversible character of flows and forces of an open system. Here, linear non-equilibrium thermodynamics is mostly discussed as it is the most advanced. We also review attempts to incorporate the mechanisms of a process into some formulations of nonequilibrium thermodynamics. The formulation of linear non-equilibrium thermodynamics for facilitated transport and active transport, which represent the key processes of coupled phenomena of transport and chemical reactions, is also presented. The purpose of this review is to present an overview of the application of non-equilibrium thermodynamics to bioenergetics, and introduce the basic methods and equations that are used. However, the reader will have to consult the literature reference to see the details of the specific applications. (C) 2002 Elsevier Science B.V. All rights reserved.
\end{abstract}

Comment: This paper was originally published in the Journal of "Biophysical Chemistry", 97 (2002) 87-111, 2002 C of this paper belongs to Elsevier. 


\section{Introduction}

It is believed that an evolved and adapted biological system converts energy in the most efficient manner for transport of substances across a cell membrane, the synthesis and assembly of the proteins, and muscular contraction. In this process the source of energy is adenosine triphosphate (ATP), which has been produced by oxidative phosphorylation (OP) in the inner membrane of the mitochondria. This is a coupled-membrane bound process. Classical thermodynamics analyzes the interconversion of energy for systems in equilibrium and provides a set of inequalities describing the direction of change. Systems may exhibit two different types of behavior: (i) the tendency towards maximum disorder; or (ii) the spontaneous appearance of a high degree of organization in space, time and/or function. The best examples of the latter are dissipative systems at non-equilibrium conditions, such as living systems. As living systems grow and develop, outside energy is needed for organized structures for the ability of reproduction and surviving in changing conditions [1].To maintain a state of organization requires a number of coupled metabolic reactions and transport processes with mechanisms controlling the rate and timing of the life processes. As Schrodinger proposed that these processes appear to be at variance with the second law of thermodynamics, which states that a finite amount of organization may be obtained at the expense of a greater amount of disorganization in a series of interrelated (coupled) spontaneous changes [1-5]. Many physical and biological processes occur in non-equilibrium, open systems with irreversible changes, such as the transport of matter, energy and electricity, nerve conduction, muscle contractions, and complex coupled phenomena. Kinetic equations and statistical models can describe such processes satisfactorily. However, it has been argued that these procedures often require more detailed information than is available, or only sometimes obtainable [6-9]. After the inspiring work and discussions of Onsager, Prigogine and Schrodinger a non-equilibrium thermodynamics (NET) approach emerged to replace the inequalities of classical thermodynamics with equalities in order to describe biological processes quantitatively [2,4,5,10-17].

There exist a large number of 'phenomenological laws' describing irreversible processes in the form of proportionalities, such as Fick's law between flow of a substance and its concentration gradient, and the mass action law between reaction rate and chemical concentrations or affinities. When two or more of these phenomena occur simultaneously in a system, they may couple and cause new effects such as facilitated and active transport in biological systems. In active transport a substrate can be transported against the direction imposed by its electrochemical potential gradient. If this coupling does not take place, such 'uphill' transport would be in violation of the second law of thermodynamics. As explained by NET theory, dissipation due to either diffusion or chemical reaction can be negative, but only if these two processes couple in an anisotropic medium and produce a positive total dissipation.

The linear non-equilibrium thermodynamics (LNET) theory is valid for near equilibrium systems in which the Gibbs free energy change is small that is $\Delta G<<2.5 \mathrm{~kJ} / \mathrm{mol}[2,3,17]$, and linear relationships exist between flows and forces identified in the dissipation function. The dissipation function is obtained from the Gibbs relation and the general transport equations of mass, momentum, energy and entropy balances of a multicomponent system. After properly identifying the forces and flows, the matrix of cross coefficients of the linear phenomenological 
equations becomes symmetric according to the Onsager reciprocal relations. The cross coefficients naturally relate the coupled flows. Through coupling a flow can occur without or against its conjugate force. This theory does not require knowledge of mechanisms of the biological process, while a complete analysis requires a quantitative description of the mechanisms of energy conversion. In practice, it is impossible to describe all the enzymes involved and the thermodynamic limitations of the possible process pathways in a kinetics formulation [18]. Therefore, network thermodynamics [19] and rational thermodynamics [20] have also been used, and they will be briefly discussed later.

Can we analyze the coupled processes in bioenergetics by a phenomenological approach using the LNET theory? This question has been discussed since the publications of Kedem and Katchalsky [21], Kedem [22], and Kedem and Caplan [23] of the analyses of transport problems in biological membranes by the LNET theory with the Onsager relations. In order to address this question, we first present a short description of the mitochondria and energy transduction in the mitochondrion. Second, LNET theory and the coupling between reactions and the transport of substances are presented. We also summarize the discussions of the proper pathways and the study of multi-inflection points that justify the use of the LNET in bioenergetics. Third, we present the concept of thermodynamic buffering caused by soluble enzymes. Besides LNET, other thermodynamic approaches namely, network thermodynamics, and rational thermodynamics are briefly summarized. Lastly, some important processes of bioenergetics are presented using the LNET formulation.

\section{Background}

\subsection{Irreversibility}

Consider the equations that describe time-dependent physical processes. If these equations are invariant to the algebraic sign of the time, the process is said to be a reversible process; otherwise it is an irreversible process. For example, the equation describing the propagation of waves in a non-absorbing medium is invariant to the substitution - $t$ for $t$; hence, the propagation of waves is a reversible process. However, Fick's equation of diffusion is not invariant with respect to time, and it describes an irreversible process. Most of the physicochemical and biological processes are irreversible processes. Phenomenological laws, which are asymmetric in time control irreversible processes. All natural processes proceed towards an equilibrium state, thus dissipating their driving force.

The word irreversibility also refers to the directedness of time evolution of a system; irreversibility implies the impossibility of creating a state that evolves backward in time. The arrow of time is related to the unidirectional increase of entropy in all natural irreversible processes.

\subsection{Energy conservation and conversion in mitochondria}

Typical mitochondria are approximately $2-3 \mathrm{~mm}$ long and $0.5-1 \mathrm{~mm}$ wide, and have an outer membrane and a folded inner membrane w24x. The membranes are constructed with tail-to-tail bilayers of phospholipids into which various proteins are embedded. Outer and inner membranes 
produce two separate compartments, the intermembrane space (C-side) containing enzymes, and the matrix (M-side) rich in proteins, enzymes and fatty acids enclosed by the inner membrane. The inner membrane, 60-70 $\mathrm{A}^{\circ}$ thick, has subunits on its very large inner surface area and recently, Mannella [25] reported new insights for the internal organization of mitochondria. Inner membrane fragments may reform into vesicles known as submitochondrial particles, which are covered by subunits. The subunits have the major coupling factors F 1 and F 0 protein parts, which together comprise the large ATPase protein complex. ATPase can catalyze the synthesis and the hydrolysis of ATP, depending on the change of electrochemical potential of proton $\Delta \mu^{-} H$. Three-dimensional images show that inner membrane involutions (cristae) have narrow and very long tubular connections to the intermembrane [25]. These openings lead to the possibility that lateral gradients of ions, molecules, and macromolecules may occur between the compartments of mitochondria. The compartment type of structure may influence the magnitude of local $\mathrm{pH}$ gradients produced by chemiosmosis, and internal diffusion of adenine nucleotides. The information on the spatial organization of mitochondria is important to understand and describe the bioenergetics. Mitochondria cause the interactions between the redox system and the synthesis of ATP, and are referred to as 'coupling membranes' [26]. The membrane is an efficient and regulated energy-transducing unit as it organizes the electron transfer and the associated reactions leading to ATP synthesis. Photosynthetic energy conservation occurs in the thylakoid membrane of plant chloroplasts. Bioenergetics is concerned with energy metabolism in biological systems [4, 17, 24], which compromise the mechanism of energy coupling [27-29] and control [30-32] within cells. The cluster of orthologous gene database identifies 210 protein families involved in energy production and conversion; they show complex phylogenetic patterns and cause diverse strategies of energy conservation [33]. The respiration chain generates energy by the oxidation of reducing equivalents of nutrients (nicotinamide adenine nucleotides NADH and the flavin nucleotides FADH 2), which is conserved as ATP through OP. Cytochrome $c$ oxidase, terminal enzyme of the chain, (i) reduces dioxygen to water membrane, 60-70 A thick, has subunits on its with four electrons from cytochrome c and four protons taken up from the matrix of mitochondria, and (ii) pumps protons from the matrix into the intermembrane space, causing an electrochemical proton gradient across the inner membrane, which is used by ATPase to synthesize ATP [34]. There is also another cycle (the Q cycle) around the cytochrome $b c 1$ complex, which causes substantial proton pumping. Synthesis of ATP is an endergonic reaction and hence, conserves the energy released during biological oxidation-reduction reactions. Photosynthesis, driven by light energy, leads to production of ATP through electron transfer and photosynthetic phosphorylation. Hydrolysis of 1 mole of ATP is $\mathrm{pH}$ and $\left[\mathrm{Mg}^{+2}\right]$-dependent, and is an exergonic reaction releasing $31 \mathrm{~kJ} / \mathrm{mol}$ at $\mathrm{pH} 7$. This energy drives various energy-dependent metabolic reactions and the transport of various ions such as $\mathrm{H}^{+}, \mathrm{K}^{+}$and $\mathrm{Na}{ }^{+}[33-39]$.

Animals adjust the energy demands by coupling of respiration to the rate of ATP utilization, efficient use of nutrients under starvation, degradation of excess food, and control of ATP production and response to stress conditions w34x. Experiments indicate that the ratio of ATP produced to the amount of oxygen consumed, which is called the $\mathrm{P} / \mathrm{O}$ ratio, changes in the range of $1-3$, and is characteristic of the substrate undergoing oxidation and the physiological organ role w26x. In the case of excess substrate, oxygen and inorganic phosphate, the respiratory activity of the mitochondria is controlled by the amount of ADP available. In the controlled state 
referred to as state 4 of the mitochondria, the amount of ADP is low. With the addition of ADP, the respiratory rate increases sharply; this is the active state called the state 3 . The ratio of the state 3 to state 4 respiratory rates is known as the respiratory control index.

Mitochondria are the major source of reactive oxygen species through the respiratory chain. These oxygen radicals may affect the function of the enzyme complexes involved in energy conservation, electron transfer and OP [40].

\section{Non-equilibrium thermodynamics (NET)}

The theory of NET provides the working equations for describing irreversible, nonequilibrium systems. For those systems not far away from equilibrium the theory of LNET, and for those systems far away from equilibrium the theory of extended non-equilibrium thermodynamics (ENET) have been developed over the last five decades. The theories of LNET and ENET appear often in modern physics, related to keywords such as non-equilibrium, motion, irreversibility, instability and dissipative structures. Prigogine and his school have studied extensively the unification of an irreversible phenomenological macroscopic description, and a microscopic description determined by linear and reversible quantum laws. Unstable phenomena occurring macroscopically in a physical system are caused by inherent fluctuations of the related state variables. The trend towards equilibrium is distinguished by asymptotically vanishing dissipative contributions. In contrast, non-equilibrium states can amplify fluctuations, and any local disturbance can move a system into unstable, metastable and structured macroscopic states. This difference is an important indication of the qualitative disparity between equilibrium and non-equilibrium states [3, 41, and 42]. The use of LNET and ENET in bioenergetics is summarized in the following sections.

\subsection{Linear non-equilibrium thermodynamics (LNET)}

The systems that are not in thermodynamic equilibrium are non-homogeneous systems in

which at least some of the intensive parameters are functions of time and position. However, a local thermodynamic state exists in small volume elements at each point in a non-equilibrium system. These volume elements are so small that the substances in them can be treated as homogeneous with a sufficient number of molecules for the phenomenological laws to apply; they may be thought of as being in thermodynamic equilibrium, and the specific entropy and specific internal energy may be determined at every point in the same way as for substances in equilibrium. Experiments show that the postulate of local thermodynamic equilibrium is valid if the gradients of intensive thermodynamic functions are small, and their local values vary slowly in comparison with the relaxation time of the local state of the system [2, 10, 12, 13, 42-47].

The entropy change $\mathrm{d} S$ for an open system is given by the following equation

$$
\mathrm{d} s=\mathrm{d} S_{e}+\mathrm{d} S_{i}=\mathrm{dQ} / \mathrm{T}+\left(\mathrm{d} S_{e}\right)_{\text {matter }}+\mathrm{d} S \quad \text { (1) }
$$


Which has two contributions: (i) the entropy flow due to exchange with the environment dSe; and (ii) The entropy production inside the volume $\mathrm{d} S i$. The value of $\mathrm{d} S e$ may be positive, negative or zero. The increment $\mathrm{d} Q e$ shows the amount of heat exchanged with the environment. Entropy production inside the elementary volume caused by irreversible phenomena is the local value of the sum of entropy generation processes. By the second law of thermodynamics, $\mathrm{d} S i$ is always positive, which is considered to be the only general criterion of irreversibility w46x. The explicit calculation of $\mathrm{d} S i$ is essentially the basis of NET theory.

Mass flow and the chemical reaction rate (called the 'flows' $\mathrm{Ji}$ ) are caused by the forces $X i$, which are the electrochemical potential gradient (for ions) $X i=$-grad $\mu i$, and the chemical affinity, respectively. Chemical affinity $A$ is

$$
A_{j}=-\sum_{i=1}^{n} \nu_{i j} \mu_{i}
$$

where $v i j$ is the stoichiometric coefficient of the $i$ th component in the $j$ th reaction, and $n$ is the number of components in the reaction. Conventionally, the stoichiometric coefficients are positive for products and negative for reactants (i.e. for a reaction $B 1+2 B 2 \leftrightarrow B 3$, the affinity would be $A=-[\mu 3-(\mu 1+2 \mu 2)])$. Generally, any force can produce any flow $J i(X i)$, and the flows and forces are complicated non-linear functions of one another. However, we can expand the non-linear dependence of the flows $J i$ and the forces $X i$ in Taylor series about the equilibrium to obtain

$$
\begin{aligned}
& f_{i}=f_{i-11}\left(X_{i}=0\right) \\
& +\sum_{j=1}^{n}\left(\begin{array}{c}
\partial J_{i} \\
\partial X_{j}
\end{array}\right)_{\mathrm{sq}} X_{j}+\frac{1}{2 !} \sum_{j=1}^{n}\left(\begin{array}{l}
\partial^{2} J_{i} \\
\partial X_{j}^{2}
\end{array}\right)_{\mathrm{eq}} X_{j}^{2}+\ldots . \\
& X_{i}=X_{i, e q}\left(A_{k}=0\right) \\
& +\sum_{i=1}^{n}\left(\begin{array}{l}
\partial X_{i} \\
\partial J_{k}
\end{array}\right)_{\circ q} J_{k}+\sum_{2}^{1} \sum_{k=1}^{n}\left(\begin{array}{c}
\partial^{2} \boldsymbol{X}_{i} \\
\partial J_{k}^{2}
\end{array}\right)_{\odot q} J_{k}^{2}+\ldots
\end{aligned}
$$

If we neglect the higher order terms, Eqs. (3) and (4) become linear relations, resulting in the general type of linear-phenomenological equations for irreversible phenomena

$$
\begin{aligned}
& J_{i}=\sum_{i=1}^{n} L_{i k} X_{i} \quad(i, k-1,2, \ldots, n) \\
& X_{i}=\sum_{k=1}^{n} K_{i k} J_{k}
\end{aligned}
$$

Eq. (5) shows that forces are the independent variables, and any flow is caused by 
contributions for all the forces, while Eq. (6) indicates that flows are the independent variables, and any force is caused by all the flows in the system. If one of the flows vanishes, Eq. (5) should to be used, while Eq. (6) is appropriate if one of the forces is set to zero. Eq. (6) may also be practical to use since it is generally easy to measure flows. The coefficients $L_{i k}$ and $K_{i k}$ are the conductance and resistance phenomenological coefficients, respectively, and are generally assumed to be time invariant. The coefficients with the repeated indices relate the conjugate forces and flows, while the cross coefficients $L_{i k}$ with $i \neq k$ represent the coupling phenomena. The phenomenological coefficients are expressed as

$$
\begin{aligned}
& L_{i k}=\left(\frac{\partial J_{i}}{\partial X_{k}}\right)_{X_{j}}=\left(\frac{J_{i}}{X_{k}}\right)_{X_{j=0}}(i \neq k) \\
& K_{i k}=\left(\frac{\partial X_{i}}{\partial J_{i}}\right)_{J_{i}}=\left(\frac{X_{i}}{J_{k}}\right)_{I_{i}=0}
\end{aligned}
$$

Since $\mathrm{d} S \mathrm{~S}>0$, the phenomenological coefficients for a two-flow system obey the following relations

$$
L_{11}>0 ; L_{22}>0 ; 1 L_{11} L_{22}-\left(L_{12}+L_{21}\right)^{2}>0
$$

Which are also valid for $K_{i j}$. For the matrix $L_{i j}$ is to be positive and definite, its determinant and all the determinants of lower dimension, obtained by deleting rows and columns, must be positive. The phenomenological coefficients are not a function of the thermodynamic forces and flows; on the other hand, they are functions of the local state and of the substance $[10,13]$. The Onsager reciprocal relations state that, provided the flows and forces are identified by the appropriate dissipation function, the matrix of phenomenological coefficients is symmetric. Onsager's relations have been proven to be an implication of the property of 'microscopic reversibility', that is the symmetry of all mechanical equations of motion of individual particles with respect to time $t$ [13]. Gambar and Markus [45] related the Onsager reciprocal relations to the global gauge symmetries of the Lagrangian. This means that the results are general and valid for an arbitrary process. In coupled processes $L_{i k}$ are not zero; for example, for a twodimensional matrix, $L_{12}$ and $L_{21}$ are numerically the same, although their physical interpretations differ.

The dissipation function, first derived in 1911 by Jaumann [48], can be obtained from the general balance equations (mass, momentum, energy and entropy) and the Gibbs relation

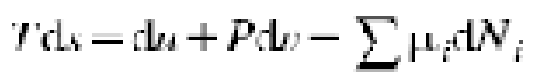

where $u$ is the specific energy, $v$ the specific volume, $P$ the pressure, $\mu_{i}$ the chemical potential and $N_{i}$ the mole number of component $i$. As the Gibbs relation is a fundamental relation of 
thermodynamics, and valid even outside thermostatic equilibrium, the entropy depends explicitly only on energy, volume and concentrations. Any changes in a process can be taken into account through the balance equations and the Gibbs relation [2, 10, and 13]. In NET theory, the entropy generation rate $\mathrm{F}$ and the dissipation function $\mathrm{C}$ are calculated as the sum of the products of the conjugate forces and flows for a specified process

$$
\Psi=T \mathbf{D}_{s}=\sum_{i=1}^{n} J_{i} X_{i} \geq 0
$$

In a stationary state, it has been shown that the total entropy production reaches a minimum, which is the stability criterion of a stationary state [2, 3, 49, and 50].

By introducing the linear phenomenological Eq. (5) into the dissipation function [Eq. (11)], we have

$$
\boldsymbol{\Psi}=\sum_{i, k-1}^{n} L_{i k} X_{i} X_{k}>0
$$

This equation shows that the dissipation function is quadratic in all the forces. In continuous systems, the choice of reference system (i.e. stationary, mass average velocity, etc.) for diffusion flow affects the values of the transport coefficients and the entropy production due to diffusion $[10,13]$. Prigogine [2] proved the invariance of the entropy production for an arbitrary base of reference if the system is in mechanical equilibrium and the divergence of viscous stress tensor vanishes.

The dissipation function for a series of $l$ chemical reactions (including the electron transport $[51,52])$ is given by

$$
\Psi=\sum_{i, k=1}^{i} J_{r i} A_{i} \geq 0
$$

For an elementary reaction the flow $J_{r}$ and the affinity $A$ are expressed in terms of forward $r_{f}$ and backward $r_{b}$ reaction rates

$$
\begin{aligned}
& J_{r}=r_{f}-f_{b} \\
& A=R I \ln \frac{r_{f}}{r_{b}}
\end{aligned}
$$

These equations are solved together to express the flow

$$
J_{r}=r_{d}\left(1-e^{A, N I}\right)
$$

Close to the thermodynamic equilibrium, where $A / R T<<1$, we can expand Eq. (16) as 


$$
f_{r}=r_{f, \mathrm{ad}} \frac{A}{R T}
$$

And compare with the linear phenomenological equations for chemical reactions

$$
J_{r i}=\sum_{i j=1}^{l} L_{i j} A_{j}
$$

to obtain the phenomenological coefficient as

$$
I_{u}=\frac{r_{j \cdot \bullet: 3 j}}{R T}
$$

Here, we have $r_{f, \text { eq }}=r_{b \text {,eq }}$. For an overall reaction with $l$ intermediate reactions, the linear phenomenological law is valid if every elementary reaction satisfies $A / R T<<1$ and the intermediate reactions are fast and hence, a steady state is reached [3].

The LNET approach is more advanced than ENET, and widely recognized as a useful phenomenological theory for describing the coupled phenomena without the need for a detailed mechanism $[5,9,35,50,53-61]$.

\subsection{Proper pathways}

Formulation of the relationships between forces and flows is the most important step in the theoretical and experimental analysis of biological reactions and transport processes [4]. This step will lead to understanding the change of affinity of an oxidative reaction driving transepithelial active transport, tissue anisotropy (compartmentalization), free energy, and activity coefficients. Experiments show that biological processes take place in many steps, each of which is thought to be nearly reversible, and exhibit linear relationships between steady state flows and conjugate thermodynamics forces, such as transepithelial active $\mathrm{Na}^{+}$and $\mathrm{H}^{+}$transport, and $\mathrm{OP}$ in mitochondria $[4,15,29,58,62,65-69]$.

Conventional phenomenological equations of NET may constitute an incomplete description of the processes, because the forces can be controlled in a proper pathway leading to near linear force- flow relationships so that the theory of LNET can be applied [4]. In this case a distinction must be made between thermodynamic linearity and kinetic linearity. For example, the flow of a solute across a membrane depends on its chemical potential and also on its thermodynamic state on both sides of the membrane. For a first order reaction $S^{\mathrm{TM}} P$, doubling the concentrations of $S$ and $P$ will double the reaction rate for an ideal system, although the force affinity remains the same. Similarly the constancy of phenomenological coefficients $L$ may be assured by the appropriate constraints to vary the force $X$ in the relationship $J=L X$. The phenomenological coefficient $L$ will reflect the nature of the membrane and be the means to vary the force

$X$. If a homogeneous thin membrane is exposed at each surface to the same concentration of the 
substances, flow is induced solely by the electric potential difference, and $L$ is constant with the variation of $X$. However, if $X$ is the chemical potential difference, dependent upon the bath solute concentrations, then $L$ becomes

$L=\frac{\psi w c_{m}}{\Delta z}$

Where $\mathrm{u}$ is the mobility, $\alpha$ is the solvent membrane partition coefficient, and $\mathrm{cm}$ is the logarithmic mean bath concentration, $\Delta c / \Delta l n c$. If some value $\mathrm{cm}$ is chosen and the concentrations are then constrained to the locus $\Delta c=\left(\mathrm{c}_{\mathrm{m}}\right) \Delta \operatorname{lnc}$, then $L$ will be constant. Kedem and Katchalsky [21] had also used the logarithmic mean concentration in the linearization in the NET formulation of membrane transport. If the force is influenced by both the concentrations and the electrical potential difference, then $L$ becomes more complex, yet it is still possible to obtain a constant $L$ by measuring $J$ and $X$ in a suitable experiment [5].

For a first order chemical reaction $S^{\mathrm{TM}} P$, the flow is given by Eq. (16)

$$
J_{r}=k_{j} c_{s}-k_{b} c_{p}=k_{b} c_{p}\left(e^{A / K I}-1\right)
$$

where $k_{f}$ and $k_{b}$ are the rate constants for forward and backward reactions, respectively, and $c$ is the concentration. At a steady state, far away from equilibrium, we describe the reaction by

$$
J_{,}=L A=L^{\prime} A / R T
$$

where $L^{\prime}=T R L$ and can be evaluated my measuring $J r$ and $A$

$$
L^{\prime}-k_{b} c_{P}\left(\frac{A / R T}{e^{A / R T}-1}\right)^{-1}
$$

Eq. (21) shows that for different values of $A$ of various stationary states, the same values of $L 9$ will describe the chemical reaction by choosing the concentrations appropriately. For a specified value of $A$, Eqs. (16) And (21) determine the concentration ratio $c_{p} / c_{s}$ and the value of $c p$, respectively. This procedure can be used to find a constant $L$ by limiting the $c_{p}$ and $c_{s}$ to an appropriate locus. As the system approaches equilibrium, $A$ tends to vanish and $k_{c} b_{p}$ approaches the value $L^{\prime}$. For a coupled process defined in Eqs. (5) and (6), we can consider a reference steady state far from equilibrium with the given forces $X_{1}$ and $X_{2}$. Proper pathways can be identified in the neighborhood of this steady state by varying the forces $X_{1}$ and $X_{2}$ in such a manner to lead to linearity of flows and forces [4, 15, 29, 58, 65-69]. Sometimes non-linearity occurs at the kinetic level because of feedback regulation and not, as is usually assumed, by large affinities that introduce thermodynamic non-linearity and hence, sustained oscillations may occur near equilibrium [70]. 


\subsection{Multiple inflection points}

Rothschild et al. [71] found the existence of a multidimensional inflection point well outside of equilibrium in the force-flow space of enzyme-catalyzed reactions, indicating linear behavior between the logarithm of reactant concentrations and enzyme-catalyzed flows. Thus, enzymes operating near this multidimensional point may lead to some linear biological systems. This range of kinetic linearity may be far from equilibrium. The conditions for the existence of a multidimensional inflection point $\mathrm{w} 5 \mathrm{x}$ are: (i) each reactant with varying activity influences the transition rates for leaving one state only; (ii) the kinetics of the transition involving the given reactant are of fixed order with respect to that reactant; and (iii) for each possible combination of reactants whose concentrations are varied, at least a certain cycle is present containing only that combination and no others. The first condition excludes autocatalytic systems; however, for many biological energy transducers it may well be satisfied. Caplan and Essig [5] provided a simple model of active ion transport, having properties consistent with the existence of a multidimensional inflection point when one of the variables was the electrical potential difference across the membrane. A multiple inflection point may not be unique; other conditions may exist where flows $J 1$ and $J 2$ simultaneously pass through an inflection point on variation of $X 1$ with constant $X 2$, and vice versa. In this case, the Onsager relations may not be valid. However, highly coupled biological systems approximately satisfy the Onsager relations [5].

Existence of the multiple inflection point may lead to proper pathways, for which linear flowforce formulation prevails. Stucki [58] demonstrated that in mitochondria, variation of the phosphate potential, while maintaining the oxidation potential constant, yields linear flow-force relationships. Extensive ranges of linearity are found for the reaction driving active sodium transport in epithelial membranes, where the sodium pump operates close to a stationary state with zero flow $[36,54]$. In the vicinity of such stationary state, kinetic linearity to a limited extent simulates thermodynamic linearity at the multidimensional inflection point. There may be a physiological advantage in the near linearity and reciprocity for a highly coupled energy transducer at the multiple inflection point, since local asymptotic stability is guaranteed by these conditions [4, 72-77]. This could be achieved, for example by the thermodynamic regulation (buffering) of enzymes, and could be interpreted as meaning that intrinsic linearity would have an energetic advantage and may have emerged as a consequence of evolution [78,79].

\subsection{Coupling in bioenergetics}

Coupling implies an interrelation between flow $J i$ and flow $J j$ so that, for example a substance can flow without or against its conjugate driving force [5, 35, 80, 81]. Coupling is in most cases due to enzymes, which catalyze two non-spontaneous processes, and make the exchange of energy between these processes possible. Tanford [27] reviewed the three kinds of coupled processes: (i) uphill transport of ions across a membrane in which an electrochemical potential gradient is created and maintained by coupling to an exergonic chemical reaction, such as ATP hydrolysis; (ii) the downhill transport of ions to drive an endergonic chemical reaction, such as ATP synthesis; and (iii) uphill transport of one type of ion coupled to the downhill transport of a second type of ion (incongruent diffusion). The first two of these are known as active transport, in which the transport of a substance is coupled to a chemical reaction, although the transported 
substance itself does not undergo chemical transformation. A general feature of active transport is that free energy coupling may involve a protein-mediated linkage between chemical changes occurring some distance apart. The need to couple the electrochemical potential change of the transported ion to translocation from one side of the membrane to the other is conceptually a more difficult process of free energy transfer without direct contact.

In mitochondria, energy-yielding reactions are coupled to outward proton translocation, while the energy-consuming reactions are coupled to the inward movement of protons [28]. In the analysis of bioenergetics the $\mathrm{H}^{+}$electrochemical potential difference acts as the coupling intermediate between the redox driven $\mathrm{H}^{+}$pumps on one side, and ATPase $\mathrm{H}^{+}$pumps on the other. The primary (respiratory chain) and secondary (ATPase $\mathrm{H}^{+}$) free energy transducing enzyme complexes can couple transmembrane proton flow to scalar chemical reactions. These primary and secondary pumps are also coupled. Westerhoff et al. [63] proposed that protonmotive free-energy coupling occurs as an array of independent, small free-energy coupling units operating as proton microunits. Electron transfer from respiratory chain substrates to molecular oxygen causes translocation of protons, and this proton motive force is utilized for ATP synthesis, ion translocations, and protein importation catalyzed by discrete multisubunit enzyme complexes located in the mitochondrial inner membrane. Hatefi [28] suggested that the synthesis of enzyme-bound ATP from enzyme-bound ADP and P does not require energy, and the substrate binding (an energy promoted process) and product releasing processes are the energy-requiring steps in OP.

Numerous studies of the relationships between flows and conjugate forces have helped to describe coupling properties of the OP pathway. A recent study by Rigoulet et al. [15] showed that the LNET approach in intact cells might be helpful for understanding the mechanisms by which OP activity is changed. They showed that with a given main substrate, the ATPconsuming processes or proton flows, lead to a unique and quasi-linear relationship between the respiratory rate and its associated overall thermodynamic driving force. The LNET approach has the following advantages: (i) behavior of OP may be studied in more relevant and physiological conditions; (ii) it is possible to study the effect of some drugs on OP, whose effects are not direct and of which the mechanism of action is unknown; and (iii) the observations in situ may be more valuable compared with studies on isolated mitochondria. The generalized force of chemical affinity for a chemical reaction shows the distance from equilibrium of the $i$ th reaction
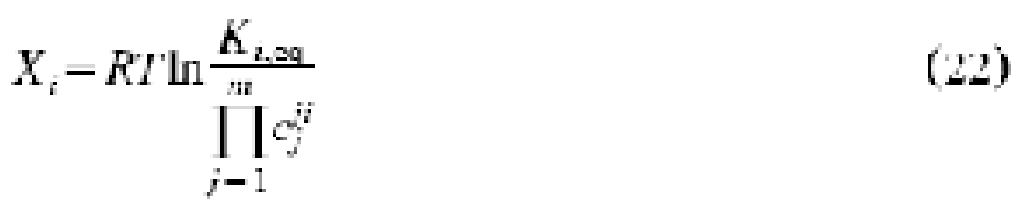

where $R$ is the gas constant, $K$ the equilibrium constant, $c_{j}$ the concentration of the $j$ th chemical species and $v_{j i}$ the stoichiometry of the $j$ th species in the $i$ th reaction. The phosphate potential in mitochondria is expressed as [58]

$$
X_{i}=-\Delta G_{p}^{0}-R^{\prime} \ln \left[\Lambda^{\prime} \mathrm{IP} /\left(\Lambda \mathrm{O} \mathrm{P}^{*} \mathrm{P}_{!}\right]\right.
$$


Stucki [58] suggested that the equilibrium thermodynamic treatment of OP is limited, and confined to the forces with vanishing net flows, hence, vanishing entropy production. To understand the OP both the forces and the flows should be considered. Also, the definition of efficiency from classical thermodynamics is not sufficient to determine the efficiency of mitochondria. In state 4 where the net ATP production is zero, the mitochondria still consume energy to maintain the phosphate potential and for the transport of ions across the inner membrane. Therefore, the assumption of thermodynamic equilibrium and hence, the vanishing of entropy production is not justified. However, the mitochondria is an open thermodynamic system not far away from equilibrium, and experiments indicate that the formalism of LNET provides a quantitative description of linear energy conversions and the degree of coupling for various output characteristics of $\mathrm{OP}[5,26,58]$. As living organisms must maintain structured states, internal entropy production must be transferred to the environment. Von Stockar and Liu [11] reviewed the entropy export and analyzed it based on the Gibbs energy dissipation that leads to microbiological growth [82-84]. For efficient growth, a high rate of biochemical coupling to exergonic catabolic processes is necessary. This implies an efficient energy conversion in which the Gibbs energy dissipation due to chemical reaction is the driving force for growth of an organism.

Stucki [58] applied the LNET theory of linear energy converters to the process of OP without considering the complex, coupled enzyme mechanisms, with the coupling expressed by the cross-phenomenological coefficients $L_{i j}(i \# j)$. Using this concept it is possible to assess the OP with $\mathrm{H}_{2 \mathrm{q}}^{+}$pumps as a process driven by respiration by assuming that the transport processes of ions $\left(\mathrm{Ca}^{2 \mathrm{q}}, \mathrm{H}^{\mathrm{q}}\right)$ are at steady state. The linear phenomenological relations are then given as

$$
\begin{aligned}
& J_{1}=I_{11} X+I_{r_{12}} X_{2} \\
& J_{2}=I_{12} X+I_{22} X_{2}
\end{aligned}
$$

where $J 1$ is the net flow of ATP, $J_{2}$ the net flow of oxygen, and $X_{1}$ the phosphate potential as given by Eq. (23), and $X_{2}$ is the redox potential, which is the difference in redox potentials between electron accepting and electron-donating redox couples. Stucki [58] and later Cairns et al. [26] experimentally showed the approximate linearity of reactions in OP within the range of phosphate potentials of practical interest.

The degree of coupling [23] is defined as

$$
q=\frac{L_{12}}{\left(L_{11} L_{22}\right)^{1 / 2}} \quad 0<|q|<1
$$

And indicates the extent of overall coupling of the different reactions driven by respiration in the mitochondria. By defining the phenomenological stoichiometry (the phenomenological stoichiometry $Z$ differs from the molecular stoichiometry n for $|q|<1$ [52]) 


$$
Z=\left(\frac{L_{11}}{I_{22}}\right)^{1 / 2}
$$

and by dividing Eq. (24) by Eq. (25), we obtain the flow ratio $j=J_{1} /\left(J_{2}\right)$ in terms of the force ratio $x=X_{1} Z / X_{2}$ as follows

$$
j=\frac{x+q}{q x+1}
$$

Since the oxidation drives phosphorylation, $X_{1}<0, X_{2}>0$, and $J_{1} / J_{2}$ is the conventional $\mathrm{P} / \mathrm{O}$ ratio, while $X_{1} / X_{2}$ is the ratio of phosphate potential to the applied redox potential. There are two types of stationary states, $s_{1}$ and $s_{2}$, considered in the analysis: (i) $s_{1}$ is analogous to an open circuit cell in which the net rate of ATP vanishes (static head); and (ii) $s_{2}$ is analogous to a close circuit cell in which the phosphate potential vanishes (level flow). These are two extreme cases, and in most practical cases neither $X_{1}$ nor $J_{1}$ vanishes, which could be experimentally realized by putting a load (the 'hexokinase trap') on to OP [52].In the state $s_{1}$ the rate of oxygen consumption $J_{2}$ and the force $X_{1}$ (the phosphate potential) are expressed in terms of the degree of coupling as follows

$$
\begin{aligned}
& \left(J_{2}\right)_{s_{1}}=L_{22} X_{2}\left(1-q^{3}\right) \\
& \left(X_{1}\right)_{s_{1}}=-\frac{q X_{2}}{Z}
\end{aligned}
$$

Where $L_{22}$ may be interpreted as the phenomenological conductance coefficient of the respiratory chain. Therefore, energy is still converted and consumed by the mitochondria. In the stationary state $s_{2}$ the flow ratio are given by

$$
\left(\frac{J_{1}}{J_{2}}\right)_{r_{3}}-{ }_{9} 7
$$

Eq. (31) shows that the $\mathrm{P} / \mathrm{O}$ ratio is not equal to the phenomenological stoichiometry $Z$, but approaches this value within a factor of $q$ if the force is kept zero. Therefore, if the degree of coupling $q$ is known, it is possible to calculate $Z$ from the $\mathrm{P} / \mathrm{O}$ measurements. 
The efficiency of linear energy converters is defined in terms of the degree of coupling and given as

$$
\mathrm{\eta}=-\frac{J_{1} X_{1}}{J_{7} X_{7}}=-\frac{x+q}{q+(1 / x)}
$$

The efficiency reaches an optimum value between the two stationary states $s_{1}$ and $s_{2}$, which is a function of the degree of coupling only, and is given by

$$
\eta_{m+t}=\frac{\psi^{2}}{\left[1+\left(\sqrt{1-q^{2}}\right)\right]^{2}}
$$

The value of $x$ at $\eta_{\text {opt }}$ is given by

$$
x_{\mathrm{op}:}=-\frac{q}{1+\sqrt{1-q^{2}}}
$$

The dissipation function, Eq. (12), can be expressed in terms of force ratio $x$ and degree of coupling $q$

$$
\frac{\Psi}{T}=\left(x^{2}+2 y x+1\right) J_{2} X_{2}^{2}
$$

If we assume $X 2$ as constant, the dissipation function has a minimum in state $s 1$ at the force ratio $x_{s l}=-q$. For linear phenomenological equations, the theorem of minimal entropy generation or the dissipation $\psi$ at steady state is a general evolution and stability criterion. Minima of $\psi$ occur along the loci of state $s 1$, and these loci are given by

$$
\frac{T_{s_{1}}}{T}-\left(1-x^{2}\right) L_{22} X_{2}^{2}
$$

The dissipation at the state of optimal efficiency is obtained using $x_{\mathrm{opt}}$ in Eq. (34), and we have

$$
\frac{\Psi_{y}}{T}=\frac{\left(1-x^{2}\right)^{2}}{1+x^{2}} L_{22} X_{2}^{2}
$$

The dissipation for state $s_{2}$ is given by 


$$
\Psi_{r_{3}}^{T^{\prime}}=L_{22} X_{2}^{\prime}
$$

Stucki [58] predicted the following order among the dissipation functions with an ATP flow $\left(\psi_{\text {opt }}\right.$ and $\left.\psi_{s 2}\right)$ and one without an ATP flow $\left(\psi_{s 1}\right)$

$$
\Psi_{v 1}<\Psi_{\text {tI I I }}<\Psi_{x_{2}}
$$

This inequality means that the minimum dissipation does not occur at the state of optimal efficiency of OP.

The dissipation of OP with an attached flow corresponding to hydrolysis of ATP is given as

$$
\Psi_{c}^{r}=J_{1} X_{1}+J_{2} X_{2}+J_{3} X_{3}
$$

Assuming that the ATP hydrolysis process is driven by the phosphate potential, $X_{3}=X_{1}$, and a linear relation exists between the net rate of ATP hydrolysis and $X_{1}$, we have

$$
J_{3}-L_{33} X_{1}
$$

Here, $L_{33}$ is an overall phenomenological conductance coefficient lumping the conductance of all the ATP utilization processes, and the dissipation function in terms of the force ratio $x$ becomes

$$
\frac{\Psi_{r}}{T}=\left\lfloor x^{2}\left(1+\frac{L_{33}}{L_{11}}\right)+2 q x+1\right] I_{20} X_{3}^{2}
$$

If these conductance $L_{33}$ and $L_{11}$ (the phenomenological conductance of phosphorylation) are matched, the following equation is satisfied

$$
\frac{L_{33}}{L_{11}}=\sqrt{1-q^{2}}
$$

Then Eq. (42) is minimum at $x_{\text {opt }}$ [26,58]. Stucki [58] viewed Eq. (43) as conductance matching of OP, which was experimentally verified for the case of perfused livers.

The dissipation function at the state of optimal efficiency of OP is 
$\frac{\left(\Psi_{c}\right)_{\text {spt }}}{T}=\frac{1-x^{2}}{1+x^{2}} l_{2,} x^{2}$

and the $\mathrm{P} / \mathrm{O}$ ratio is given by

$$
\left(\frac{J_{1}}{J_{2}}\right)_{\text {opt }}=\left(\frac{J_{1}}{J_{2}}\right)_{s_{2}} \frac{1+x^{2}}{2}
$$

This equation shows that unless the coupling is complete $(q= \pm 1)$, a maximal $\mathrm{P} / \mathrm{O}$ ratio is incompatible with optimal efficiency, and the following inequality occurs $[26,58]$

$$
\frac{1}{2}\left(\frac{J_{1}}{J_{2}}\right)_{v}<\left(\frac{I_{1}}{I_{2}}\right)_{3 x}<\left(\frac{I_{1}}{I_{2}}\right)_{x_{2}}
$$

Therefore, experimentally measured low $\mathrm{P} / \mathrm{O}$ ratios do not necessarily mean a poor performance of the OP. Similarly, the net rate of ATP synthesis at optimal efficiency is given as

$$
\left(J_{1}\right)_{e y t}=\left(J_{1}\right)_{x_{2}} \frac{1-x^{2}}{2}
$$

With the limits

$$
\left.0<i, l_{2}\right)_{\text {opt }}<\frac{1}{2}\left(J_{1}\right)_{s 2}
$$




\begin{tabular}{|c|c|c|c|}
\hline $\begin{array}{l}\text { Prondurtion } \\
\text { finctian }\end{array}$ & $\begin{array}{l}\text { I Ni of the nprinal } \\
\text { efinie-cy mores. }\end{array}$ & $q$ & $\begin{array}{l}\text { rnermy } \\
\text { cret } \\
\text { sincil-nut }\end{array}$ \\
\hline 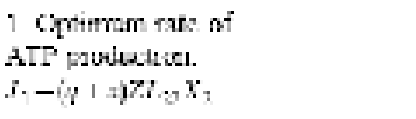 & 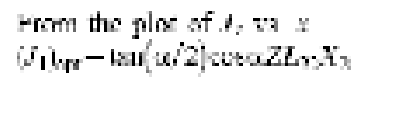 & $\begin{array}{l}q_{t}=1 ; \mathrm{ins} \\
x-51.83\end{array}$ & $n=\operatorname{sen} \alpha$ \\
\hline 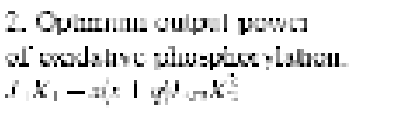 & 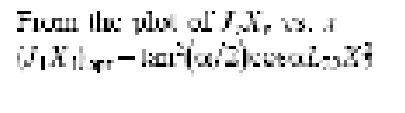 & $\begin{array}{l}a_{-}-0.910 \\
x-65.53\end{array}$ & $2-\cos s$ \\
\hline 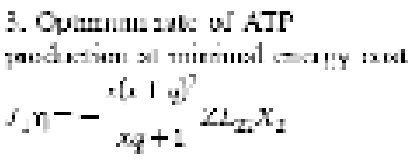 & 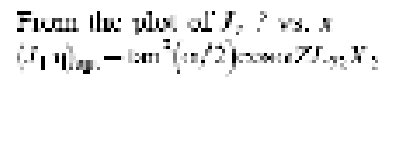 & $\begin{array}{l}4 y-0.253 \\
2-7738^{f}\end{array}$ & Yx: \\
\hline 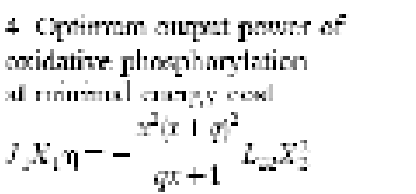 & 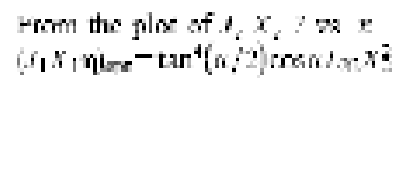 & 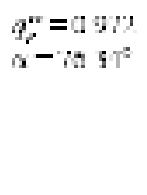 & res \\
\hline
\end{tabular}

Indicating that a maximal net rate of ATP production is incompatible with optimal efficiency.

Stucki [58] analyzed the required degree of coupling of OP, which satisfies Eq. (43), for the optimum production functions $f$ for ATP and output power expressed in terms of $\alpha=\arcsin (\mathrm{q})$

$$
f=\tan ^{m}(\alpha / 2) \cos (\alpha) \quad(m=1,2,3,4)
$$

The optimum production functions and the associated constants are described in Table 1, while Fig. 1 shows the effect of degree of coupling on the characteristics of four different output functions $f$. If the system has to maximize the ATP production at optimal efficiency then $f=$ $\left(J_{1}\right)_{\text {opt }}$ and $q_{\mathrm{f}=0.786}$.

Instead, if the system has to maximize the power output at optimal efficiency, we have the output function $f=\left(J_{1} X_{1}\right)_{\text {opt }}$. occurring at $q_{p}=0.91$.

If the additional constraint of efficient ATP synthesis (minimal energy cost) is imposed on these output functions, then the economic ATP flow and economic power output occur at $q_{\mathrm{f}}{ }^{\mathrm{ec}}=0.953$ and $q_{\mathrm{p}}{ }^{\mathrm{ec}}=0.972$, respectively. The difference between $q p$ and $q_{\mathrm{p}}{ }^{\text {ec }}$ becomes clear when we calculate the output power, and the product of power output and efficiency at the maximum of the plots of $\left(J_{1} X_{1}\right)_{\text {opt }}$ (from the plot of $J_{1} X_{1}$ vs. $x$ ) and $\left(J_{1} X_{1} \eta\right)_{\text {opt }}$ (from the plot of $J_{1} X_{1} \eta$ vs. $x$ ). A transition from $\mathrm{q}_{p}$ and $q_{\mathrm{p}}{ }^{\text {ec }}$ causes a $12 \%$ drop in output power $\left(J_{l} X_{l}\right)$ and $51 \%$ increase in efficiency [58]. Cairns et al. [26] compared the theoretical and observed determinations of coupling of OP in mitochondria from rat liver, heart, and brain using classical and NET measures; the coupling of OP can change with substrate availability and also reflect the specific response of mitochondria to fit specific organ roles in the rat. For example, the metabolic driving force and flow (ATP production rates) relationships facilitated the analysis of $\mathrm{Ca}^{2+}$ effects on various commonly accepted control points within OP. 
Stucki w85x analyzed the sensitivity of the force, the phosphate potential, to the fluctuating cellular ATP utilization, and found that the sensitivity is minimal at a degree of coupling $q=0.95$. This is based on an eigenvalue sensitivity analysis of the experimentally supported LNET model of OP, and indicated that the phosphate potential is highly buffered with respect to changing energy demand, and the value of $q$ agrees with the degree of coupling at which net ATP production of OP occurs at optimal efficiency. This leads to simul

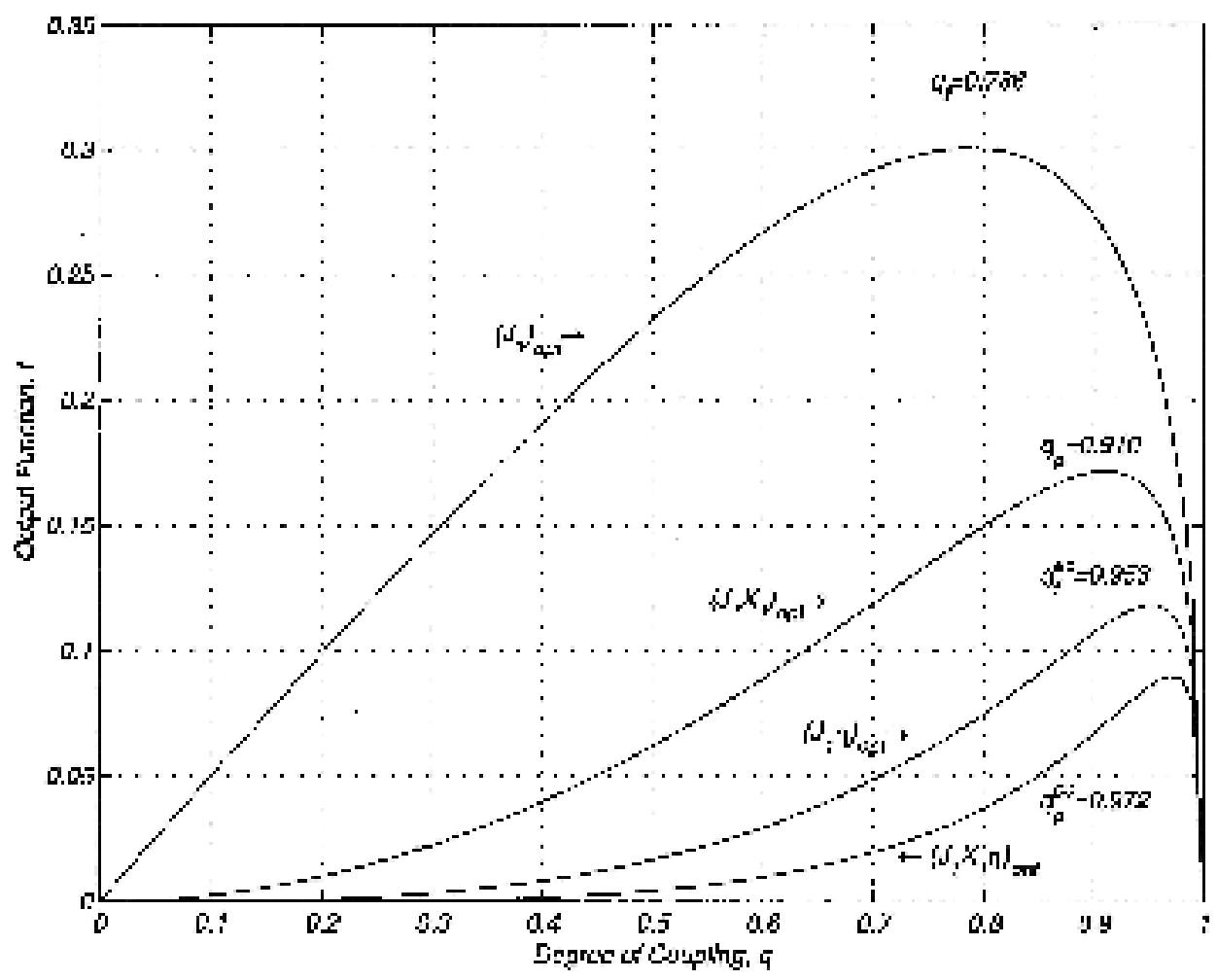

Fig. 1. Degree of couplings and different output functions $f$.

taneous maximization of kinetic stability and thermodynamic efficiency at the same degree of coupling. For $\mathrm{H}^{\mathrm{q}}$-translocating ATPase, the $\mathrm{H}^{\mathrm{q}}$ /ATP coupling ratio is important for mechanistic, energetic and kinetic consequences, and a value of four was adopted for the ratio.

The values of the degree of coupling depend on the nature of the output required from the energy conversion system in the mitochondria [30, 60, 64, 86-92]. Tomashek and Brusilow [93] suggested that the differences in rates of proton pumping, ATPase activities, and degrees of coupling might all be variables in the choices that each biologic system makes in order to survive and compete in its environment. Experiments with liver perfused at a metabolic resting state suggest that Eq. (43) is satisfied over a time average, and the degree of coupling $q^{e c}$ yields an efficient process of OP [58]. Optimization may be carried out based on other constraints different from the efficiency. For example, the production of thermal energy in the mitochondria requires rather low degrees of coupling. Pfeffier et al. [65] showed that ATP production with a low rate and high yield results from cooperative resource use and may evolve in spatially 
structured environments. The thermodynamically determined degrees of coupling were also measured in $\mathrm{Na}$ transport in epithelial cells [55], and growing bacteria [84] where maximization of net flows are the most important task of the system. On the other hand, for a fed rat liver in a metabolic resting state, economy of power output has the priority, while a starved rat liver has to produce glucose, and the priority of energy conversion is replaced by the maximum ATP production. Theoretical and experimental studies indicate that the degree of coupling is directly related to metabolic regulation and stability in living organisms $[26,58,86]$. If the system cannot cope with instabilities, then fluctuations such as $\mathrm{pH}$ and pressure of the blood could irreversibly harm the organism.

\subsection{Thermodynamic regulation in bioenergetics}

Fatty acids may regulate and tune the degree of coupling [76,87-89], and can induce uncoupling to set the optimum efficiency of OP $[88,90]$. Experiments with incubated rat-liver mitochondria show that the adenylate kinase reaction can buffer the phosphate potential to a value suitable for optimal efficiency of OP in the presence of very high rate of ATP hydrolysis. Stucki [76] called this class of enzymes, such as adenylate kinase and creatine-kinase, thermodynamic buffer enzymes. A fluctuating ATP/DP ratio and deviations from optimal efficiency of OP are largely overcome by thermodynamic buffering [54, 57, and 91]. The enzymes are capable of causing certain reaction pathways by catalyzing a conversion of a substance or a coupled reaction [17, 18, 31, and 92]. For example, on adding nigericin to a membrane with gradients of $\mathrm{H}^{+}$and $\mathrm{K}^{+}$, the system reaches a steady state in which the gradients of $\mathrm{H}^{+}$and $\mathrm{K}^{+}$are balanced. On the other hand, if we add valinomycin and protonophore, both gradients rapidly dissipate. Wyss et al. [29] discussed the functions of the mitochondrial creatine kinase, which is a key enzyme of aerobic energy metabolism [87,94,95], and involved in the buffering, transport and reducing the transient nature of the system in reaching a new steady state upon changes in workload [68]. This can be achieved: (i) by increasing the enzymatic activities in such a way as to guarantee near equilibrium conditions; (ii) by metabolic channeling of substrates; and (iii) by damping oscillations of ATP and ADP upon sudden changes in workload.

Uncoupling proteins facilitate the dissipation of the transmembrane electrochemical potentials of $\mathrm{H}^{+}$or $\mathrm{Na}$ produced by the respiratory chain, and results in an increase in the $\mathrm{H}^{+}$and $\mathrm{Na}$ permeability of the coupling membranes. These proteins provide adaptive advantages, both to the organism and to individual cell, and also increase vulnerability to necrosis by compromising the mitochondrial membrane potential [88, 96, and 97]. Some uncoupling is favorable for the energy-conserving function of cellular respiration $[86,98]$. LNET is also used for a macroscopic description of some inhibitors of OP, by considering protonophores, some ATPase inactivators and some electron-chain inhibitors [99].

In mitochondrial OP leaks cause a certain uncoupling of two consecutive pumps, for example electron transport and ATP synthase, and may be described as membrane potential driven backflow of protons across the bilayer, while a slip means a decreased $\mathrm{H}^{+} / \mathrm{e}^{-}$stoichiometry of proton pumps [32]. Kadenbach et al. [34] recently proposed that mitochondrial energy metabolism was regulated by the intramitochondrial ATP/ADP ratio and slip of proton pumping in cytochrome $c$ oxidase at high proton motive force. In transportation, leaks, for example, can 
be found in the proton-sugar symport in bacteria where a protein mediates the transport of protons and sugar across the membrane, and adding a protonophore, a parallel pathway occurs causing a leak in the transport. A slip can occur when one of two coupled processes (i.e. reactions) in a cyclic process proceeds without its counterpart, which is also called intrinsic uncoupling [93]. Leaks and slips may affect the metabolic rate [100,101]. Schuster and Westerhoff [101] developed a theory for the metabolic control by enzymes that catalyze two or more incompletely coupled reactions. Control by the coupled reactions is distinguished quantitatively from control by the extent of slippage using the LNET formulation. Here, the limits of coupling or the tightness of coupling, as Krupka [73,74] called it, becomes an important parameter and may be defined as the ratio of coupled-to-uncoupled rates, which is a function of the binding energy of the substrate and the carrier protein. Evolution favors the coupling in organisms as tight as possible, since the loss of available metabolic energy is a disadvantage. For example, the rather loose coupling of calcium and sugar transport systems may represent a tradeoff between efficiency and the rate that can be achieved in primary and secondary active transport [74]. One other concern in an interconnected biological network is that the behavior of a subsystem (e.g. glycolysis) may become unsteady and chaotic, so that the output of this subsystem (e.g. ATP production) is adversely affected, and becomes an external noise for other subsystems causing inhibition and desynchronization [4].

\subsection{Extended non-equilibrium thermodynamics (ENET)}

Extended non-equilibrium thermodynamics is used for systems far from equilibrium. The distance from equilibrium can be measured by the gradients and affinities imposed on the system. For example, chemical reaction systems are in the linear regime if the affinities $A$ are small compared to $R T(A<2.5 \mathrm{~kJ} / \mathrm{mol}$ at $T=300 \mathrm{~K})$, however, for most reactions chemical affinities are in the range of $10-100 \mathrm{~kJ} / \mathrm{mol}$ and hence, they are in the non-linear regime. Beyond a critical distance the flows are no longer linear functions of forces; the system explores all the possibilities to counter the applied gradients, and may become unstable and have more than one possible steady state, sometimes showing bifurcation and leading to self-organized behavior. As the Belousov-Zhabotinski reaction displays, these complex structures can arise as the solution of a deterministic differential equation. Sometime it is the symmetry, and not the linearity, of the force-flow relations in the near equilibrium region that precludes oscillations [56]. The process and the boundary conditions do not uniquely specify the new non-equilibrium state, which can be a highly structured state and can only be maintained by a continuous exchange of energy and/or matter with the surroundings. The structured states degrade the imposed gradients effectively when the dynamic and kinetic pathways for the structure were not possible. ENET is concerned with the non-linear region, and with deriving the evolution equations with the dissipative flows as the independent variables in addition to the usual conserved variables [2, 20,102].

If $\psi$ is the dissipation in a non-equilibrium stationary state, the change in $\psi$ with time due to small changes in the forces $d X_{i}$ and in the flows $d J_{i}$ is expressed by 


$$
\begin{aligned}
\frac{\mathrm{d} \Psi}{\mathrm{d} t}- & \int_{V}\left(\sum J_{i} \frac{\mathrm{d} X_{i}}{\mathrm{~d} t}\right) \mathrm{d} V \\
& +\int_{V}\left(\sum X_{i} \frac{\mathrm{d}_{i}}{\mathrm{~d} t}\right) \mathrm{d} V=\frac{\mathrm{d}_{X} \Psi}{\mathrm{d} i}+\frac{\mathrm{d}_{t} \Psi}{\mathrm{d} i}
\end{aligned}
$$

In the non-linear regime and for time-independent boundary conditions we have [2, 3, and 49]

$$
\frac{\mathrm{d}_{x} \Psi}{\mathrm{d} x} \leq 0
$$

$\mathrm{d}_{X} \psi$ is not a differential of a state function, so that Eq. (51) does not indicate how the state will evolve, it only indicates that $\psi$ can only decrease. Stability must be determined from the properties of the particular steady state. This leads to the decoupling of evolution and stability in the nonlinear regime, and it permits the occurrence of new organized structures beyond a point of instability of a state in the non-equilibrium regime. The time-independent constraints may lead to oscillating states in time, such as the well-known Lotka- Volterra interactions in which the system cycles continuously.

A physical system $x$ may be described by an $n$-dimensional vector with elements of $x_{i}$ $(i=1,2, ., n)$ and parameters $a_{j}$, and

$$
\frac{\mathrm{d} x_{i}}{\mathrm{~d} t}=f,\left(x_{i}, a_{j}\right)
$$

The stationary states $x_{s i}$ are obtained using $\mathrm{d} x_{i} / \mathrm{d} t=0$. With a small perturbation $\delta x_{i}$ and a positive function $L(\delta x)$ called the distance, the stationary state is stable if the distance between $x_{i}$ and the perturbed state $\left(x_{s i}+\delta x_{i}\right)$ steadily decreases with time, that is

$$
L\left(\delta x_{i}\right)>0 ; \quad \frac{\mathrm{d} L\left(\delta x_{i}\right)}{\mathrm{d} z}<0
$$

A function satisfying Eq. (53) is called a Lyapunovfunction, which provides a general criterion for stability of a state. Kondepudi and Prigogine [3] used the second variation of entropy $L=-\delta^{2} S$ 
as a Lyapunovfunctional if the stationary state satisfies $\sum \delta \boldsymbol{X}_{i} \delta J_{j}>0$, hence, a nonequilibrium stationary state is stable if

$$
{ }_{\mathrm{d} i}^{\mathrm{d} \delta^{2} S}=\sum \delta X_{i} \delta J_{j}>0
$$

The bilinear expression in Eq. (54) is known as the excess entropy production [3], and $\mathrm{d} J_{i}$ and $\mathrm{d} X_{i}$ denote the deviations of $J_{i}$ and $X_{i}$ from the values at the non-equilibrium steady state.

The coupling between chemical kinetics and transport may lead to dissipative structures that are caused by auto-and cross-catalytic processes with positive and negative feedback, influencing their rates of reaction w56x. For example, the Belousov- Zhabotinski reaction exhibits a wide variety of characteristic non-linear phenomena. In the nonlinear region, the class of instabilities arise in biological systems are (i) multi-steady states, (ii) homogeneous chemical oscillations, and (iii) complex oscillatory phenomena [3]. The thermodynamic buffer enzymes may provide bioenergetics regulatory mechanisms for the maintenance of a state far from equilibrium [39, 86,103,104].In recent studies ENET has been used to describe protein folding and the complex behavior in biological systems $[39,103]$.

\section{Other thermodynamic approaches}

LNET has some fundamental limitations: (i) it does not incorporate mechanisms into the formulation, or provide values of the phenomenological coefficients; and (ii) it is based on the local-equilibrium hypothesis, and therefore confined to systems in the vicinity of equilibrium. Also, properties not needed or defined in equilibrium may influence the thermodynamic relations in non-equilibrium situations. For example, the density may depend on the shearing rate in addition to temperature and pressure [20]. The local equilibrium hypothesis holds only for linear phenomenological relations, low frequencies and long wavelengths, which makes the application of the LNET theory limited for chemical reactions. In the following sections, some attempts that have been made to overcome these limitations are summarized.

\subsection{Rational thermodynamics}

Rational thermodynamics (RT) provides a method for deriving constitutive equations without assuming the local equilibrium hypothesis. In the formulation, absolute temperature and entropy do not have a precise physical interpretation. It is assumed that the system has a memory, and the behavior of the system at a given time is determined by the characteristic parameters of both the present and the past. However, the general expressions for the balance of mass, momentum and energy are still used [20].

The Clausius-Duhem equation is the fundamental inequality for a single component system. The selection of the independent constitutive variables depends on the type of system considered. A process is then described by a solution of the balance equations with the 
constitutive relations and Clausius-Duhem inequality.

Rational thermodynamics is not limited to linear constitutive relations, and when the constitutive equations are expressed in terms of functionals, generally, a vast amount of information is necessary [20]. Rational thermodynamics may be useful in the case of memory effects; non-equilibrium processes may approach equilibrium in a longer time than as is generally assumed; as a result nature has a much longer memory of irreversible processes [3]. Grmela [105] combined thermodynamic theories, such as LNET, ENET, rational thermodynamics, and theories using evolution criteria and variation principles into a bracket formalism based on an extension of Hamiltonian mechanics. One result of this bracket approach is a general equation for the non-equilibrium reversible-irreversible coupling (GENERIC) formalism for describing isolated discrete systems of complex fluids [106].

\subsection{Network thermodynamics (NT)}

Flow-force relations are more complicated in bioenergetics due to enzymatic reactions; for example, Hill [107] reported many critical constraints involved in describing photosynthesis in terms of LNET [108]. Network thermodynamics (NT) and mosaic non-equilibrium thermodynamics [17] incorporate the details of the process into force-flow relations of LNET for complex systems such as biological applications. The Onsager reciprocity and topological connectedness in the network representation is of concern and discussed by Mikulecy [109]. NT can be used in both the linear and non-linear regions of NET, and has the flexibility to deal with the systems in which the transport and reactions are occurring simultaneously, either at steady or at unsteady state [109-113]. For example, in the formalism of network thermodynamics a membrane is treated as a sequence of discrete elements called lumps, where both dissipation and storage of energy may occur. Each of the storage and dissipative elements needs relationships between the input and output flows. The lumps have a resistance and capacitance, and incorporate the boundary conditions when they joined in the network.

The NT formulation has been used in the thermodynamics of bacterial growth, the steady state protonic coupling scheme $[63,114]$, and following the biological free-energy converters scheme [17]. First, bacteriorhodopsin liposomes use light as energy source to pump protons. The flowforce relations for each of the elemental processes are written and by adding the flows of each chemical substance, a set of equations is obtained based on the proposed structure of the system, so that the verification of the relations can be used to test the applicability of the proposed structure. If the relations are not verified, then either the formulation is in error or the proposed structure is not appropriate. In testing the formulation experimentally certain states, such as steady states, are assumed. For example, prediction of the effect of addition of ionophores on the rate of light-driven proton uptake is experimentally tested; the light-driven pump is inhibited by the electrochemical gradient of protons developed by the system itself. Second, the formulation of the OP is based on the chemiosmotic model, assuming that the membrane has certain permeability to protons that the ATP synthetase is a reversible $\mathrm{H}^{+}$pump coupled to the hydrolysis of ATP, and that the proton gradient $\Delta \tilde{\mu}_{\mathrm{H}}$ across the inner mitochondrial membrane is the main coupling agent. The following flow- force relations are used 


$$
\begin{aligned}
& J_{\mathrm{H}}=L_{\mathrm{H}} \Delta \tilde{\mu}_{\mathrm{H}} \\
& J_{\mathrm{O}}=I_{\mathrm{U}}\left(\Delta G_{\mathrm{U}}+\gamma_{\mathrm{H}^{n_{\mathrm{H}}}} \Delta \tilde{\mu}_{\mathrm{H}}\right) \\
& J_{\mathrm{H}}=L_{\mathrm{H}}\left(\Delta G_{\mathrm{L}^{\prime}}+\gamma_{\mathrm{H}^{n}} n_{\mathrm{H}} \Delta \bar{\mu}_{\mathrm{H}}\right)
\end{aligned}
$$

The terms $L \mathrm{H}, L_{\mathrm{O}}$ and $L_{\mathrm{P}}$ are the transport coefficients for proton, oxygen and ATP flows, respectively. The $\gamma$ factors describe the enzyme-catalyzed reactions with rates having different sensitivities to the free-energy difference for the proton pump and other reactions. This differential sensitivity is a characteristic of the enzyme and is reflected in the formulation of the flow-force relationships of that enzyme. The term $n_{\mathrm{H}}$ is the number of protons translocated per ATP hydrolyzed, while $J_{\mathrm{H}}, J_{\mathrm{O}}$ and $J_{\mathrm{P}}$ indicate the flows of hydrogen and oxygen and ATP, respectively.

Metabolic control theory can also be combined with NT in which the metabolism is lumped together in three essential steps [112]: (i) catabolism; (ii) anabolism; and (iii) leak (ATP hydrolysis without coupling to anabolism or catabolism). The LNET formulation is used in the network approach to describe the coupled diffusion of water and the cryoprotectant additive in cryopreservation of a living tissue [113,115]. Assuming that the living tissue is a porous medium, Darcy's law is used to model the flows of water and cryopreservation agent, and a temperature-dependent viscosity is used in the model. The Onsager reciprocal relations are valid, and the three independent phenomenological coefficients are expressed in terms of the water and solute permeability, and the reflection coefficient. The network thermodynamics model is able to account for interstitial diffusion and storage, transient osmotic behavior of cells and interstitium, and chemical potential transients in the tissue compartments.

\section{Some applications of linear non-equilibrium thermodynamics}

\subsection{Oxidative phosphorylation $(O P)$}

Dissipation function for OP is

$$
\Psi-J_{1^{u}} A_{1^{3}}+J_{H} \Delta \ddot{\mu}_{H}+J_{\mathrm{C}} A_{O}
$$

Here, the subscripts $\mathrm{P}, \mathrm{H}$ and $\mathrm{O}$ refer to the phosphorylation, $\mathrm{H}^{+}$flow, and substrate oxidation, respectively and $\Delta \ddot{\mu}_{\mathrm{II}}=\Delta \ddot{\mu}_{\mathrm{I}}-\Delta \ddot{\mu}_{\mathrm{Ir}}^{0}$. The dissipation function can be transformed to $[4,11]$

$$
\Psi=J_{1} A_{L^{2}}+J_{H} \Delta \ddot{\mu}_{H}+J_{U} A_{\mathcal{O}}^{e x}
$$

where $\mathrm{A}^{e x}$ is the external affinity. When the interior of the mitochondrion is in a stationary state, 
for all components $\mathrm{d} N j, i n / \mathrm{d} t=0$, hence

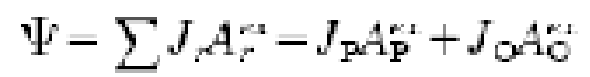

Therefore, in the stationary state it suffices to measure the changes in the external solution only.

From Eq. (59), the appropriate phenomenological equations in terms of the resistance formulations, which are suitable for considering a vanishing force, are given by

$$
\begin{aligned}
& A_{\mathrm{P}}^{e x}=K_{\mathrm{P}} J_{\mathrm{P}}+K_{\mathrm{PH}} J_{\mathrm{H}}+K_{\mathrm{pO}} J_{\mathrm{O}} \\
& \Delta \tilde{\mu}_{\mathrm{H}}=K_{\mathrm{LH}^{1} J_{1}}+K_{\mathrm{H}} J_{\mathrm{H}}+K_{\mathrm{OH}} J_{\mathrm{U}} \\
& A_{\mathrm{O}}^{\kappa_{1}}=K_{\mathrm{PO}} J_{\mathrm{P}}+K_{\mathrm{OH}} J_{\mathrm{H}}+K_{\mathrm{O}} J_{\mathrm{O}}
\end{aligned}
$$

For oxidative phosphorylation the coupling is essentially 'chemiosmotic', i.e. $K_{\mathrm{PO}} \sim 0$ [52], and there are three degrees of coupling, $q_{\mathrm{PH}}, q_{\mathrm{OH}}$ and $q_{\mathrm{PO}}$.

When the force vanishes, $\Delta \mu^{2} \mathrm{H}=0$, Eqs. (61) and (63) give

$$
\begin{aligned}
A_{\mathrm{P}}^{\kappa_{1}=} & K_{\mathrm{P}}\left(1-q_{\mathrm{PH}}^{2}\right) J_{\mathrm{P}} \\
& -\left(K_{1} K_{\mathrm{O}}\right)^{1 / 2}\left(q_{\mathrm{PO}}+g_{\mathrm{PH}} q_{\mathrm{OH}}\right) J_{\mathrm{U}} \\
A_{\mathrm{O}}^{e x}= & -\left(K_{\mathrm{P}} K_{\mathrm{O}}\right)^{1 / 2}\left(q_{\mathrm{PO}}+q_{\mathrm{PH}} q_{\mathrm{OH}}\right) J_{\mathrm{P}} \\
& +K_{\mathrm{O}}\left(1-q_{\mathrm{OH}}^{2}\right) J_{O}
\end{aligned}
$$

So that

$$
q=\frac{q_{\mathrm{PC}}+q_{\mathrm{PII}} q_{\mathrm{OTT}}}{\left(1-q_{\mathrm{PH}}^{2}\right)\left(1-q_{\mathrm{OH}}^{2}\right)}
$$

- The effectiveness of energy conversion can be expressed as

$$
\mathrm{T}=-\frac{J_{P} X_{P}}{J_{\infty} A_{\sigma}^{-19}}
$$

Where $X_{p}$ is the force for proton transportation, and $\mathrm{h}$ vanishes at states $s_{1}$ and $\mathrm{s}_{2}$.

It is also useful to consider the force developed per given rate of expenditure of metabolic energy, the efficacy of force [5]

$$
\sigma_{X P}=-\frac{X_{L^{\prime}}}{J_{r r} A_{i}^{\operatorname{arp}}}
$$


Stucki [58], and Caplan and Essig [5] provide a detailed analysis of the chemical, chemiosmotic and the parallel coupling hypothesis.

\subsection{Facilitated transport}

Many biological transport processes occur selectively and fast as a result of the combination of a substrate $s$ with a membrane constituent referred to as carrier $c$ to form a carrier-bounded complex $b c$, which shuttles between the two surfaces of a membrane (or perform conformational changes)

$n \mathrm{O} 2(s)+\mathrm{Hb}(c)$ hemoglobin $\leftrightarrow \mathrm{HbO} 2 \mathrm{n}$ oxyhemoglobin $(b c)$

For facilitated oxygen transport, a membrane composed of a filter soaked in a solution of hemoglobin was used in experimental work and formulated by LNET [9, 21]. Oxygen, at different pressures $P_{1}>P_{2}$, was placed in the two compartments and the steady state flow of oxygen across the membrane was measured. The following dissipation function is used

$$
\Psi=J g\left(-\mu_{g}\right)+J_{g} \nabla\left(-\mu_{c}\right)+J_{b e} \nabla\left(-\mu_{\varepsilon e}\right) \quad(70)
$$

and the affinity $A$ is

$$
A=n \mu_{s}+\mu_{c}-\mu_{b c}
$$

Assuming that the rate of reaction is more rapid than diffusion, so that the reaction is at equilibrium and hence, $A=0$, and applying the gradient operator to Eq. (71), we obtain

$$
n \nabla \mu_{s}+\nabla \mu_{c}=\nabla \mu_{\infty}
$$

Eq. (72) expresses a chemical interrelation among the forces that leads to a coupling of the flows. The flows passing any point in the membrane are those of free oxygen $J_{s}$, of hemoglobin $J_{c}$, and of oxyhemoglobin $J_{b c}$. The externally measured flow of oxygen $J_{\mathrm{s}}$ equals to the flows of free oxygen and oxygen carried by hemoglobin.

$$
J_{s}^{\times}=J_{s}+n J_{b c}
$$

Since no external flow of hemoglobin $\mathrm{J}_{\mathrm{c}}{ }^{*}$ takes place, we have

$$
I_{c}^{*}=I_{c}+I_{b c}=0
$$

Using Eqs. (72)-(74), we transform the dissipation Eq. (70) into

$$
\Psi=J_{s}^{*} \nabla\left(-\mu_{s}\right)+J_{c}^{*}\left(-\mu_{c}\right)
$$


The flows and forces identified in Eq. (75) are used in the following linear phenomenological equations

$$
\begin{aligned}
& J_{s}^{*}=-L_{s} \nabla \mu_{,}-L_{s} \nabla \mu_{r} \\
& J_{c}^{*}=0=-L_{c s} \nabla \mu_{s}-L_{c c} \nabla \mu_{c}
\end{aligned}
$$

Eqs. (76) and (77) obey the Onsager relations, $L_{s c}=L_{c s}$, which reduces the number of unknown phenomenological coefficients by one. The chemical potentials are related to concentrations or partial pressures. If $P 1>P 2$, then $c s, 1>c s, 2$, and the contribution of the carrier transport to the total oxygen flow is positive. The presence of hemoglobin enhanced the flow of oxygen at low oxygen pressure; however, this facilitation of oxygen transfer disappeared at higher pressures of oxygen. Hemoglobin can exist in two conformational states, differing in oxygen affinity, and all four oxygen-binding sites change their affinity simultaneously [27].

\subsection{Active transport}

For a two-flow system of diffusion and chemical reaction the dissipation function is

$$
v=T \Phi_{s}=J_{i} X_{i}+I, A \geq 0
$$

where $J_{r}$ is the rate of chemical reaction, and $J_{i}$ is the flow of a substance. Eq. (78) represents the simplest thermodynamic description of coupled flow-chemical reaction systems. If $J_{i} X_{i} i$ s negative, then the signs of $J_{i}$ and $X_{i}$ are opposite, causing the flow in a direction opposite to that imposed by its conjugate force $X_{i}$, which is called the active transport system. This is possible only if the flow is coupled to the chemical reaction with a large positive dissipation $J_{r} A$, leading to a positive over-all dissipation without contradicting the second law of thermodynamics [4, 38, 58]. Active transport is a universal property of the cells and tissues, and determines the selective transport of substrates coupled to metabolic reactions [5,9]. Most of the chloride flow in plant cells depends on continuing photosynthesis. Biological membranes are anisotropic as their molecules are preferentially ordered in the direction of the plane of the membrane, so that the coupling between chemical reactions (scalar) and diffusion flow (vectorial) can take place, which does not occur in an isotropic medium [2]. Sodium, potassium and proton pumps occur in almost all cells, especially nerve cells, while the active transport of calcium takes place in muscle cells.

Conventional methods for establishing the existence of active transport are to analyze the effects of metabolic inhibitors, to correlate the level or rate of metabolism with the extent of ion flow or the concentration ratio between the inside and outside of cells, and to measure the current needed to short-circuit a system having identical solutions on each side of the membrane. The measured flows indicate that the flow contributing to the short circuit current, and any net flows detected are due to active transport, since the electrochemical gradients of all ions are zero $\left(\Delta \|=0, r_{s}=r_{i}\right)$.

Kedem [22] presented an early analysis of the interactions between mass transport and 
chemical reactions using the LNET approach. The dissipation function for active transport may be expressed as

$$
\Psi=\sum J_{j} \Delta \tilde{\mu}_{i}+J_{i} A_{F}
$$

where $\Delta \tilde{\mu}_{\mathrm{i}}$ is the difference in electrochemical potential on two sides of the membrane, $J_{j}$ and $J_{r}$ are the flow of ions and the rate of reaction, respectively, and $A$ is the conjugate affinity within the cell. Since the flows are easy to measure, it may be advantageous to express the phenomenological equations in terms of the forces

$$
\begin{aligned}
& \Delta \dot{\mu}_{i}=\sum_{j=1}^{n} K_{j i} l_{j}+K_{i r} I^{\prime} \\
& A_{r}=\sum_{j=1}^{n} K_{r r} J_{j}+K_{r r} J_{r}
\end{aligned}
$$

The resistance cross-coefficient $K_{i r}(i \neq r)$ represents the coupling between the flow and the rate of reaction. Although the coefficient $K_{i r}$ must be a vector (since diffusion flow is a vector and the rate of reaction is scalar), flows of species transported across a membrane may be treated as scalars since their direction is affected by the topology of the system. In order to use Eqs. (80) and (81), we need to know the local flows and forces in the biomembrane system, and integrate the expressions over the membrane thickness. The rate of metabolic reaction $J_{r}$ may be taken, for example as the rate of oxygen intake by a tissue $[9,22,58$, and 81$]$. The diffusion flow rate $J_{i}$ may be obtained from Eq. (80) as

$$
J_{i}=\frac{\Delta \hat{\mu}_{i}}{K_{i i}}-\sum_{j-1, i+j} K_{i j} K_{i j} J_{j}-\frac{K_{i r}}{K_{i i}} J_{r}
$$

The driving force for the ionic flow is the electrochemical potential difference, which comprises both the differences in concentration and in the electrical potential, and for an ideal system is given as

$$
\Delta \tilde{\mu}_{i}-R T\left(\ln c_{i}^{\top}-\ln c_{i}^{\top}\right)+z_{i} F\left(\psi^{\top}-\psi^{\top}\right)
$$

Where I and II denote the surrounding compartments to the membrane.

Based on the dissipation function [Eq. (79)], the following phenomenological equations can describe a simple example for an active transport of sodium $[5,9]$ 


$$
\begin{aligned}
& \Delta \bar{\mu}_{\mathrm{N} x}=K_{\mathrm{Na}} I_{\mathrm{N} x}+K_{\mathrm{N} s}, \\
& \Delta \tilde{\mu}_{\mathrm{Cl}}=K_{\mathrm{Cl}} J_{\mathrm{Cl}} \\
& A-K_{\mathrm{Ne} e} J_{\mathrm{Na}}+K_{r} J_{r}
\end{aligned}
$$

These equations imply that no coupling exists between the sodium and chloride flows, while the sodium flow $J_{\mathrm{Na}}$ is coupled to metabolic reaction $J r$. The flow of chloride may be assumed to proceed in a passive manner.

Eqs. (84)- (86) can be applied to the following cases: (i) two electrodes are inserted into each compartment and are short circuited, the potential difference $\left(\psi_{1}-\psi_{\mathrm{II}}\right)$ is zero and an electrical current $I$ is allowed to flow across the membrane. If the experiment is carried out at equal salt concentrations in I and II, so that $(\ln c \mathrm{I}-\ln c \mathrm{II})=0$, we have

$$
\Delta \tilde{\mu}_{\mathrm{Na}}=0 . \quad \Delta \tilde{\mu}_{C 1}=0
$$

The only remaining driving force is the affinity $A$ of the metabolic reaction in Eq. (84), so that we have

$$
J_{\mathrm{Na}_{\mathrm{a}}}=-\frac{K_{\mathrm{Nar}}}{K_{\mathrm{N}_{\mathrm{A}}}} J_{r}
$$

Since the flow of electricity is determined by the ionic fluxes

$$
I=\left(J_{N_{1}}-J_{\mathrm{C}}\right) H
$$

Therefore, from Eqs. (88) and (89) we have

$$
l=-\frac{K_{\mathrm{Nar}}}{K_{\mathrm{Na}}} l, F
$$

Under short-circuit conditions, the measurable electrical current is linearly proportional to the overall rate of reaction, and the proportionality constant depends on the coupling coefficient $K \mathrm{Na} r \neq 0$. (ii) In an open circuit potentiometric case where $I=0$, a transport of salt occurs $J \mathrm{Na}=J$ $\mathrm{Cl}$.

For analysis we can express the phenomenological stoichiometry $Z$, and the degree of coupling $q$ between the sodium transport and chemical reaction as follows 


$$
\begin{aligned}
& Z=\left(\frac{K_{r}}{K_{\mathrm{VIa}}}\right)^{1 / 2} \\
& q=-\frac{K_{\mathrm{Ner}}}{\left(K_{\left.\mathrm{N}_{2} K_{r}\right)^{1 / 2}}\right.}
\end{aligned}
$$

For the general case we have for the degree of coupling $-1 \leq q \leq 1$.

While the above model is useful, biological membranes that transport various substances are more complex. Such membranes are almost composite membranes with series and parallel elements [5]. A value of $q<1$ shows an incomplete coupling, in which metabolic energy must be expended to maintain an electrochemical potential difference of sodium even if $J_{\mathrm{Na}}=0$.

\subsection{Molecular evolution}

Proteins are synthesized as linear polymers with the covalent attachments of successive amino acids, and many of them fold into a three-dimensional structure defined by the information contained within the characteristic sequence [116]. This folding results largely from an entropic balance between hydrophobic interactions and configurationally constraints. The information content of a protein structure is essentially equivalent to the configurationally thermodynamic entropy of the protein, which relates the shared information between sequence and structure [116-118]. Dewey [117] proposed that the time evolution of a protein depends on the shared information entropy $S$ between sequence and structure, which can be described with a NET theory of sequence-structure evolution; the sequence complexity follows minimal entropy production resulting in a steady non-equilibrium state $[2,3,102]$

$$
\frac{\partial}{\partial X_{j}}\left(\frac{\mathrm{d} s}{\mathrm{~d} t}\right)=0
$$

From a statistical mechanical model of thermodynamic entropy production in a sequencestructure system, a NET model was developed [118]. In the model, the shared thermodynamic entropy is the probability function that weights any sequence average; the sequence information is defined as the length of the shortest string the encodes the sequence; and the connection between sequence evolution and NET is that the minimal length encoding of specific amino acids will have the same dependence on sequence as the shared thermodynamic entropy. Dewey and Donne [117] considered the entropy production of the protein sequence-structure system based on LNET. The change of composition with time is taken as the flow, while the sequence information change with the composition is treated as the force, which can be interpreted as the chemical potential of the sequence composition. Since the change of entropy with time (dissipation) is a positive quadratic expression, [Eq. (12)], Eq. (93) shows that when regions of the sequence are conserved, the rest of the sequence is driven to a minimum entropy production and hence, towards the lower complexity seen in the protein sequence, creating a stable state away from equilibrium.

The recognition that in the steady state a system decreases its entropy production, and loses minimal amounts of free energy led to the concept of least dissipation, which is the physical 
principle underlying the evolution of biological systems [9,119]. A restoring and regulating force acts in any fluctuation from the stationary state.

\subsection{Molecular machines}

Some important biological processes resemble macroscopic machines governed by the action of molecular complexes [120-123]. For example, pumps are commonly used for the transport of ions and molecules across biological membranes, whereas the word motor is used for transducing chemical energy into mechanical work by proteins [123]. Proton translocating ATP synthase (F0F1) synthesizes ATP from ADP and phosphate, coupled with an electrochemical proton gradient across the biological membrane. Recently, rotation of a subunit assembly of the ATP synthase was considered as an essential feature of the ATPase enzyme mechanism and that F0F1 as a molecular motor [124]. These motors are isothermal, and the internal states are in local equilibrium. They operate with a generalized force, which for the motor/ filament system may be the external mechanical force $f$ ext applied to the motor and the affinity $A r$, which measures the free-energy change per ATP molecule consumed. These generalized forces create motion, characterized by an average velocity $v$, and average rate of ATP consumption $J r$. Julicher et al. [120] used the following LNET formulation

$$
\begin{aligned}
& \Psi=v f_{\mathrm{ex:}}+J_{r} A_{r} \\
& v=L_{11} f_{\mathrm{oxt}}+L_{12} A_{r} \\
& J_{r}=L_{21} f_{\mathrm{oxt}}+L_{22} A_{r}
\end{aligned}
$$

Here, $L_{11}$ is a mobility coefficient, while $L_{22}$ is a generalized mobility relating ATP consumption and the chemical potential difference, and $L_{12}$ and $L_{21}$ are the mechano-chemical coupling coefficients. A given motor/filament system can work in different regimes. In a regime where the work is performed by the motor, efficiency is defined as

$$
\eta=-\frac{v f_{\text {ext }}}{I_{r} l_{r}}
$$

For non-linear motors operating far from equilibrium velocity, reversal allows direction reversal without any need for a change in the microscopic mechanism [120]. 


\section{Conclusions}

Most physical and biological systems operate away from thermodynamic equilibrium. The distance from equilibrium distinguishes between two important non-equilibrium thermodynamic states.(i) The system is in a near-equilibrium state, and the linear non-equilibrium thermodynamics formulation with the phenomenological approach provides the working equations for coupled irreversible processes. (ii) The distance surpasses a critical value after which the system may exhibit structured states only maintained with a constant supply of energy. These states are non-linear in their flow-force relationships and sometimes are called dissipative structures. Living systems operate along the linear and non-linear regions. Especially in the last five decades, non-equilibrium thermodynamics has been used in bioenergetics and membrane transport to describe the energy conversion and coupling between chemical reaction and diffusion flows. For processes of bioenergetics not far away from equilibrium, the formulation of linear non-equilibrium thermodynamics and the use of the dissipation-phenomenological equation approach may be helpful, because it does not need a detailed description of the mechanism of the bioenergetic processes. For those processes of bioenergetics far away from equilibrium, the extended thermodynamics theory may be a helpful tool. Other practical applications of non-equilibrium thermodynamics are the rational thermodynamics and network thermodynamics in which the mechanism of the process can be incorporated in the formulation. This review does show that the theory of non-equilibrium thermodynamics can be a tool to describe and formulate the coupled phenomena of transport and chemical reactions taking place in living systems without the need of detailed mechanisms.

\section{Acknowledgments}

Y.D. thanks the Center for Molecular and Engineering Thermodynamics of the University of Delaware for the hospitality during the visit to prepare this work.

\section{References}

[1] O Tolssaint, F, D Schenider, The thermodyname and evolution of complexity in biological systems. Comp. Binchem Physiol A 120 (1998) 3-5 
[2] 1. Arizogin, Lerodaxtion to 'Lkernotyaanics of las:verskle Proresses: Wiley New York 1567

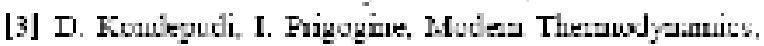
reon feat Engince to Dasipative strutues, Wilsy. Now Yurk, 1:Sy)

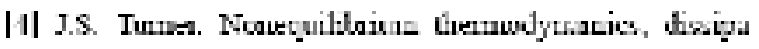

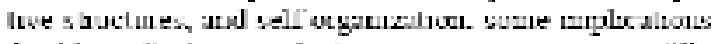

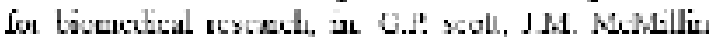
(Tds). Distupatur Strucheres ard Spanarenporal

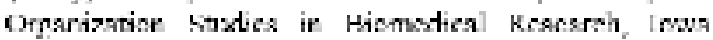
State tinwersity Press, Amer, 1975

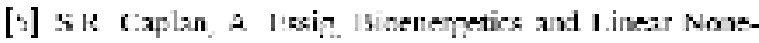

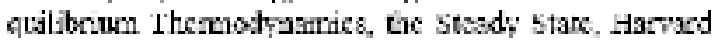
Dnnessty Press, Comihndge. ISS

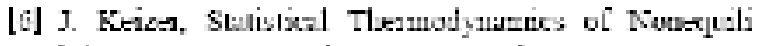
brina lYocesace, Soringer, New Yod, $198 \%$

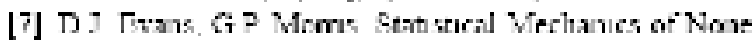

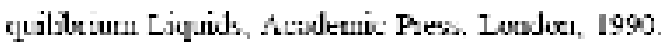

[8] B.C. En, Kinete lecory and lreversible Itecmodynam15s. Wiley. New Yark, 199?

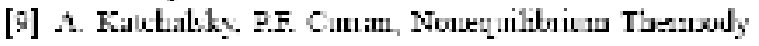

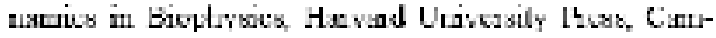
bndme, 1967

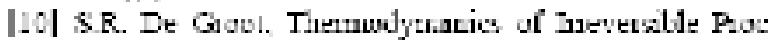

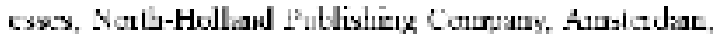
1)

[111 U. woc Stcckar, J. S. Li:, Does rrecrobual Lfe alwrys test on regatre entrogy? Thermotynamic aralysts of

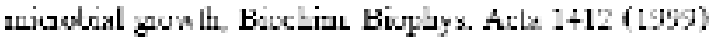
1) 13-211.

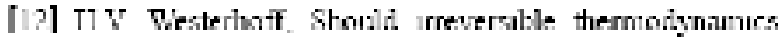

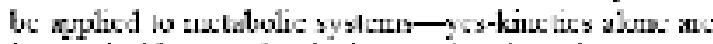

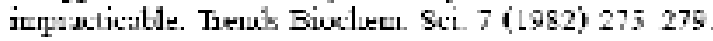

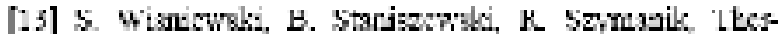

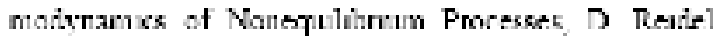

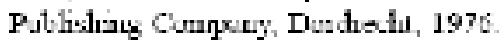

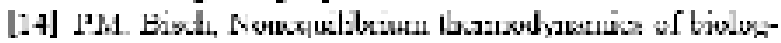
real spotens, Mras I Mat Thi Res of (1993) $41:-4$

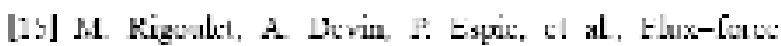

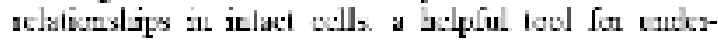
standistr, the Treingisen of axidaries pinosphorplation alterntions? Buochum Rushys Acta 1765 (1959) 117 1.1.

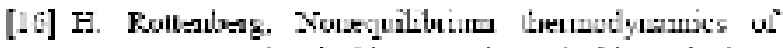
caerm? centersion in bisenergetics, Bicckin. Bioplys. Ara $549(1379) 205-253$

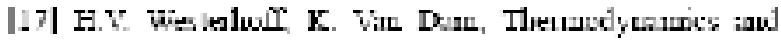

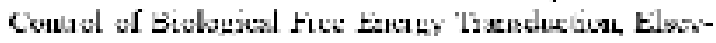
iz, Atusicaders, $198 \%$

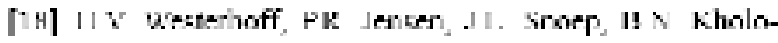
derks. Themmodynancs of canplexity the live rell. Ibeswardine Aclz 309 (1958) t:1-120.

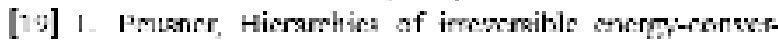

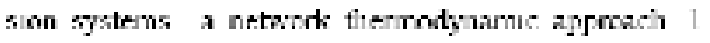

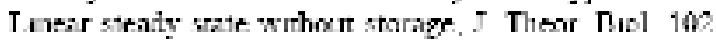
(1.983) 735 . 


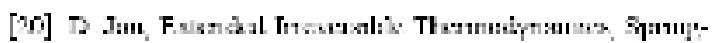
er-verlaps kes rork, 1 sen

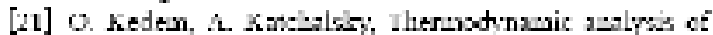

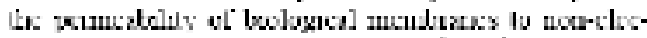
coches, Doschum. Doophss. Act2 27 (LS58) 229

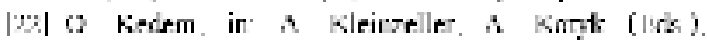

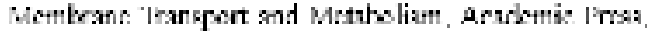
Nees rock. 1901, pg. S:.

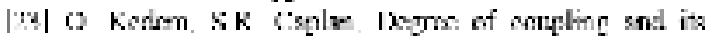

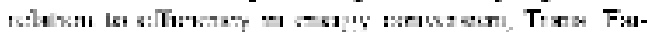
adiy Soc of (19k) 14y:

[21] C.V. Jon:a, Dublogual Duergy Conservation, Wueg, New Tak., 1970 .

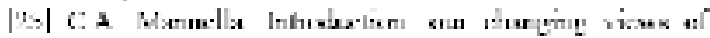

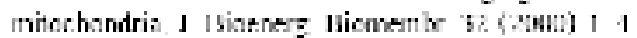

|26| CD. Cums, J. Waltber, AL. Hariser, A Daneser,

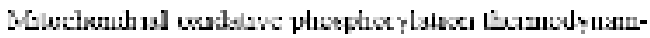

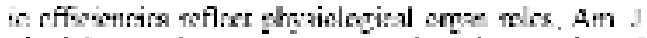

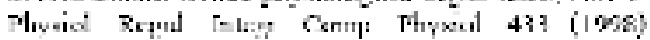
$21 \sin -16$ tis?

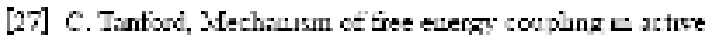
Leaspel, Auel. Rox. Buxducul 52 (1983) 379-6).

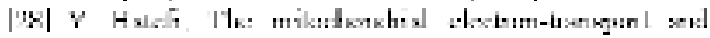

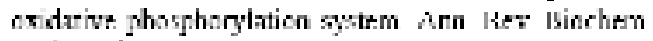

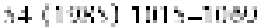

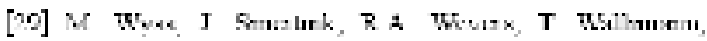

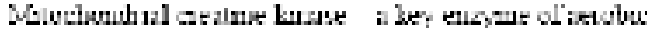

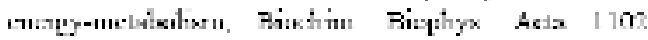
(1947) 119-106

[00] II v. Whestehet: NecequLbemm fhersadvnasuc con sideraticn of tat efficiency. ceirrol, ald regulatice of unsobulal arowth. Pree hapl Cism 55 (L9SI) 1395-190:

|i1| CiC: Broun, W 12 Hrand thematynsenic cantml of

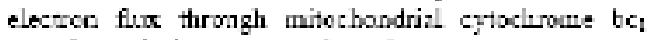

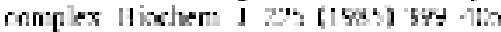

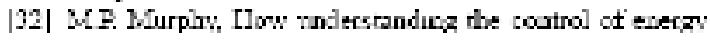

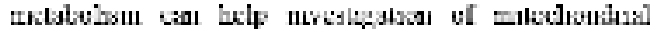

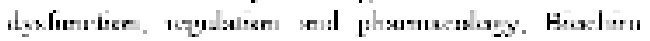

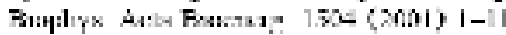

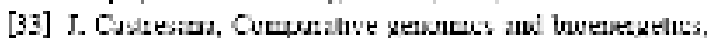
Sioclim Jispays. Acen 15v94 (2005) 110.

$|: 4|$ is Koderba:h, M I lumenarn, Si Arnald, I Iet, I:

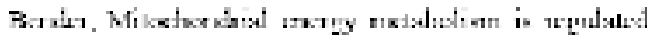
ria nowlear coded sahnanis of cyterhome $\angle$ nxidase. irse codiciL Buol. Med. 29 12000) 21.221.

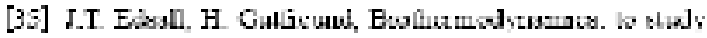

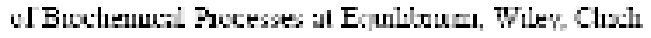
अ4.7 1983

[iم] OA Candia FS Keinsch themotyneni analytis of scuve sodum and pocassmm tracepor: in the frog egi

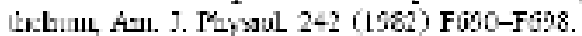

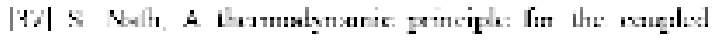

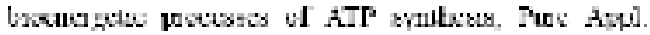
Chem io (1998) A $31-\mathrm{CH} 4$

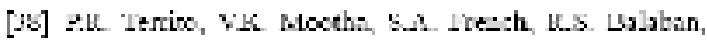

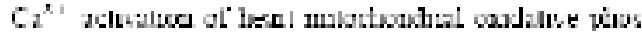

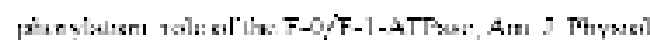

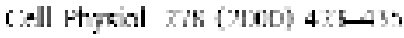

[j9] M. V. Mesquits, A.K Vascocelloa, R. Luzzi, Corpiex

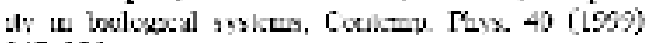
217256

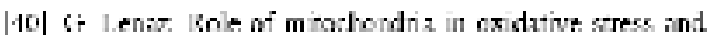

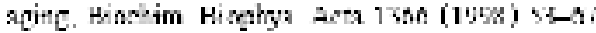

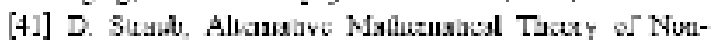

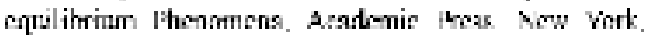
1507

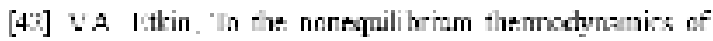
buslogucal systems, Dustintis 10 (1995) 656 676.

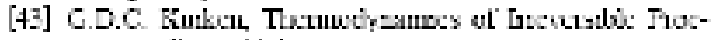

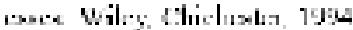

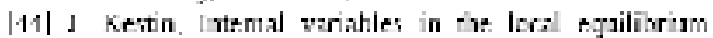
afpeorumation, J. Nou ZquLib. Thermodyz. I8 (LSSI) $3005 / 9$

$|44|$ \& Carmer. 4 Marhas, On the glahal symmetry of

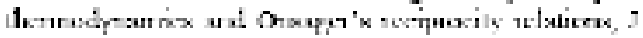

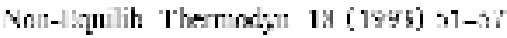

[16] Xw. Tscisez], Fundamentals of Davibavm ind

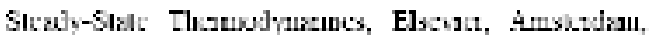
row

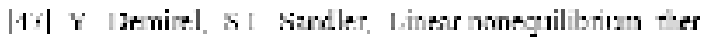

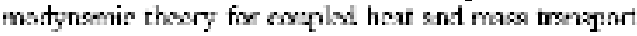
lut. J. Heat Mass Innsier 11 (2001) 2139 2151.

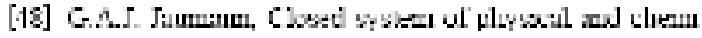

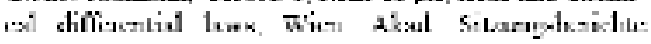

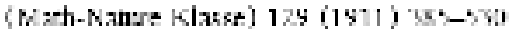

[19] 2. Glansicatr I Fngogers, Tiermodyume Thecry ot Strusture. Stability and Lhcrustions. Wiley, Nen York, $\lfloor 97 .$.

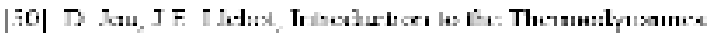
of hislogical irarsase Imentse Hsll Hndewand Clifsi, N., : 950 .

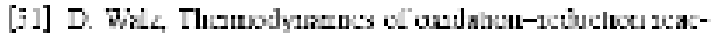
uous and its apelisation to bueneczetis, Dotim.

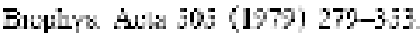

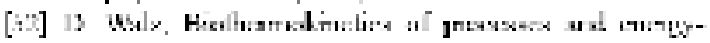
conversion. Bucchin. Lioplyx. Acto 101S (tSs) Lil 221

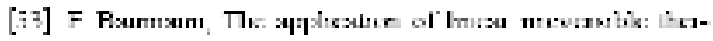

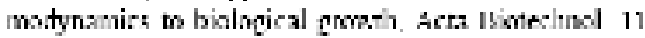
(1991) 3 5

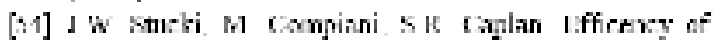
enexp converson in model bxlozical p:mps optun

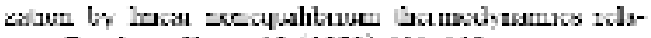

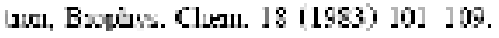

$|\$ 2|$ J. Lihar A. Lscaz, Sk. Crplan, lbecmodruawe degrte of scruling herween metahalism and sadiun-transort In frog жin, Diochum. Dusplyx. Acta 112 (1975) $350-302$.

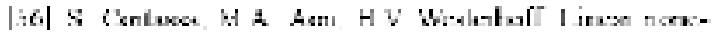

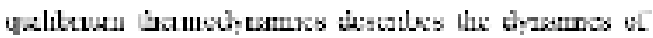

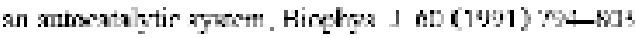

[5] J.W. soves, Optimization of mino:boudial energy cou

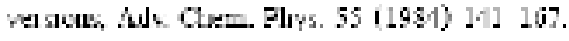


[s\&] I $W$ Struskı. The ogt mal efticiency and the erromur

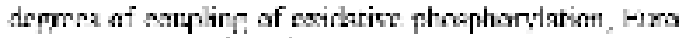
J. Duocher 109 (1500) 259293.

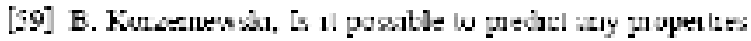
of axdituve plocphorylation m a therencal wry? . Mal Coll Bwtı: LII (1995) 315358.

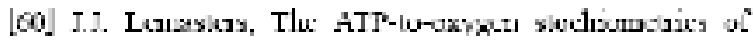

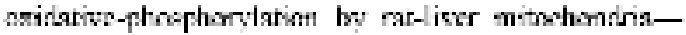

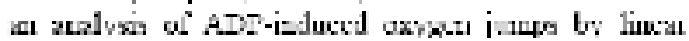

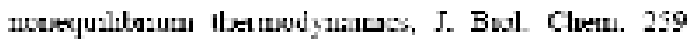
$(1984) 3123-3130$.

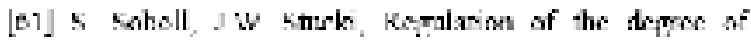

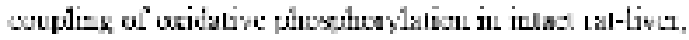

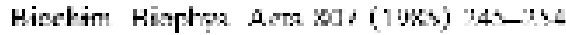

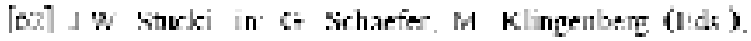

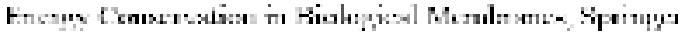
Verlaz. Terlen 1576 on 361987

[63] IIV Testerhoft, D.A. Melanis, G Ventrol. G.I.

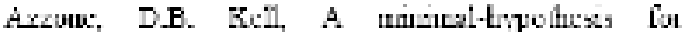
wentace labed tree eaergy trasdistwo the role of

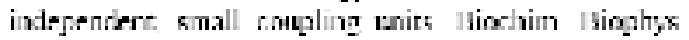
Acts 763 (19961) 257292.

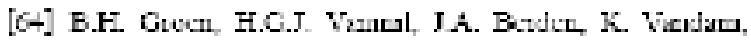
The efficirrey of exidarve-pheeptervilarion in porars-

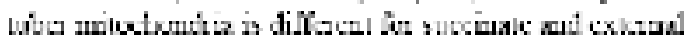
NADIE. Buochum Duophys. Acts 1.10 (1992) 37 11.

[es] I Pfelfier, si sehuster Si sanhoeffer, Cooperation and

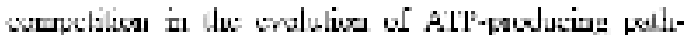
ways triense sy: (xun) no4-xi:

[66] J. Nueisen, Metatoilc control inalysas of buochenusa]

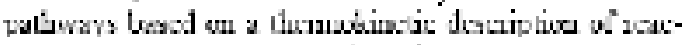
tou mten, Buchem 2. 321 4.997) 133138.

[oid] I Wagaer, $K$ lalkner if liakner, Infornation abat

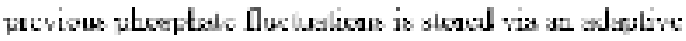

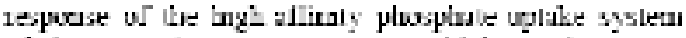

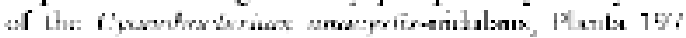
(1955) $1-7-155$.

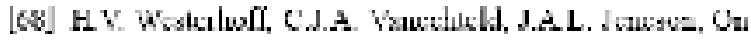
the expectest relariorshig hetween cilbue energy of Alp

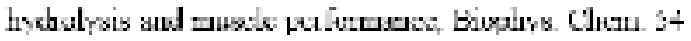
(1955) $157 \mathrm{l} / 2$.

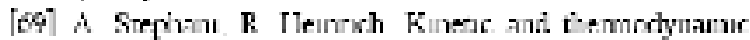
principles determining the strustaral resige of al' froduenz systeass. Ditl. Mrti. Diol. 60 (1999) $900-343$

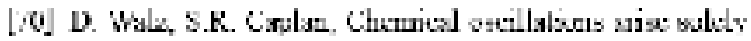

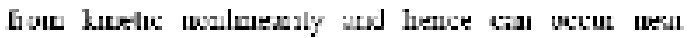
oqualibrmen, Ticphys I 69 (1995) 1658 1767

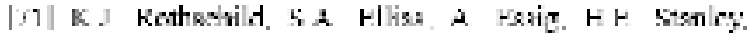

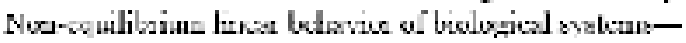
existence of exnme-nectiated mustidimentional infles-

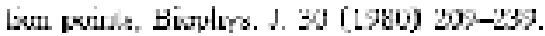

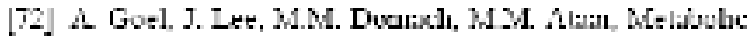

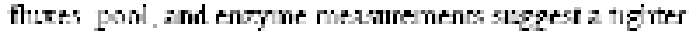

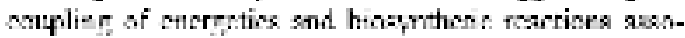
clared parh aed:ced pyrwart buase fitox, Dictst. Iticere $n+(1 \%$ y) $1: 5-1 \% 4$

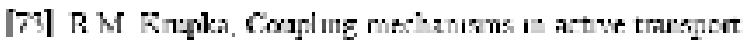

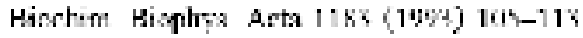

[Al] K $\mathrm{M}$ Knupkr. I.imits an the tighmes of complirg in

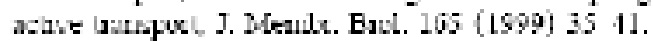

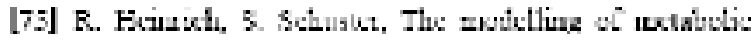
systews. Structure. coctrol and optunality, Bicsystems 4 (

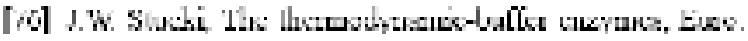
J. Buxìn 109 (1980) 257267.

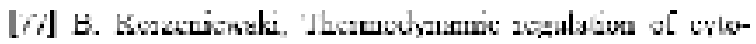
Linoter uxdise, Mul. Cell. Budentil. 171 (1997) $13 \%-141$.

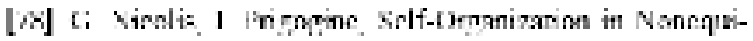
Erium Systus, Wilex New Youk 1977

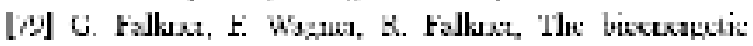

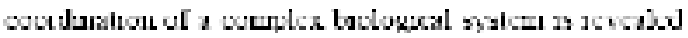

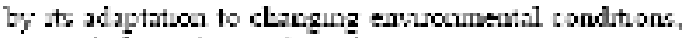
Acla Bivinouctica 44 (1996) 283-299

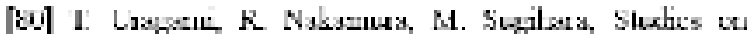

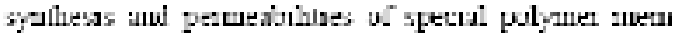
hrones, 53 drave tronsport of 1. pioerylaluoune throngh

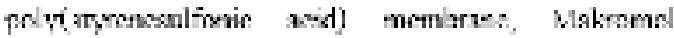
Clkan Kaysd. 33 (1582) 141-14j.

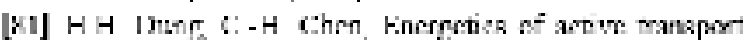

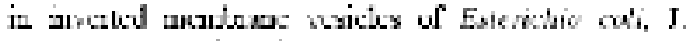
Neabe Sc1 55 (1591) 327310.

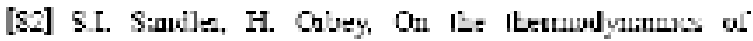

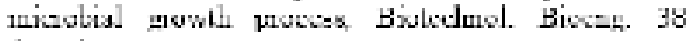
(.2.1) $007-7.3$.

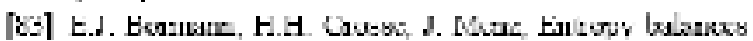

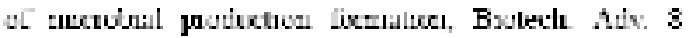
(1950) 27799

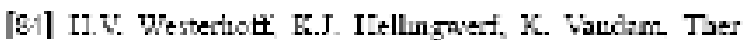

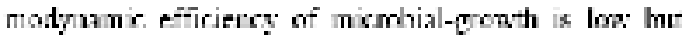

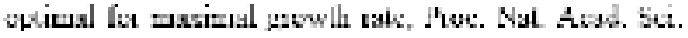
USA Bivl. Saz. B0 (1983) 305-309

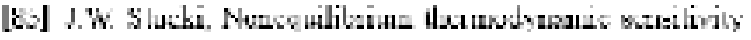

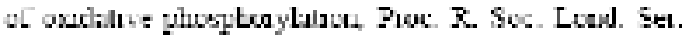
Eivi. Sci. $244(1 \% 21) 1 \% ;-21)_{2}$.

[G6] Y.R. Stuln:b:r: Lincoupluz: new approacbes to an ald

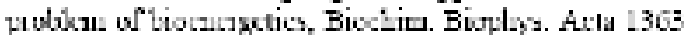
1.958) 100121.

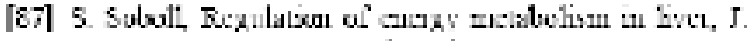
Ducenerg. Duouembs. 27 (1995) 571 582.

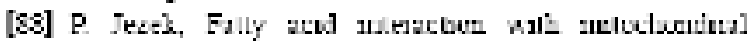

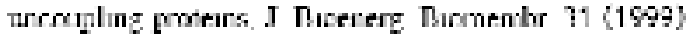
$4: 3-40$

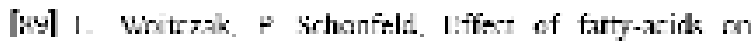

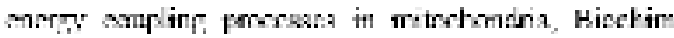
Bicṕciys. Acia 1183 (1923) -1-57.

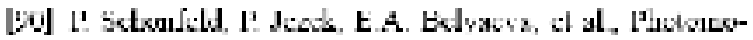

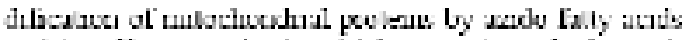

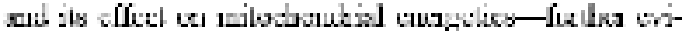

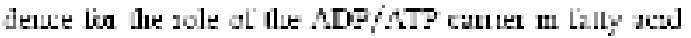
medated evcouplung. Duro. J. Dicclev. 210 (1995) $3 \mathrm{~F} ; \mathrm{i} / \mathrm{F}$ 


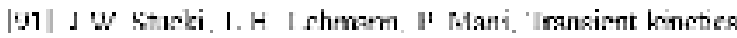

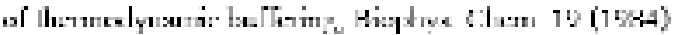
$1 ? 1115$

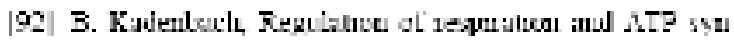
these an hugies orgneses hypothess, J. Dosearg. Dicmendbe 18 (1986) 39 니.

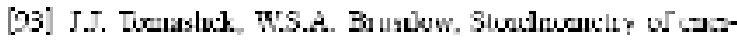

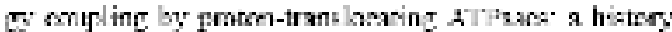

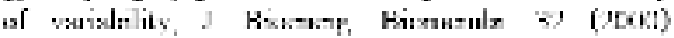
473-300.

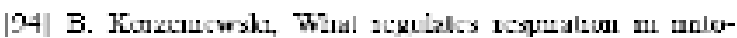

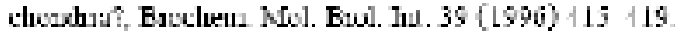

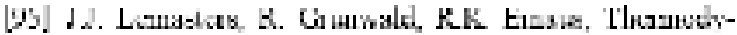

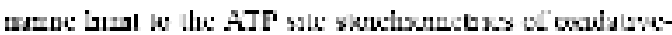
p'asphorylation by rat-lizer mitarhandrix, 」 ltisl

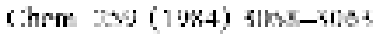

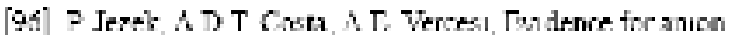

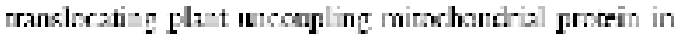

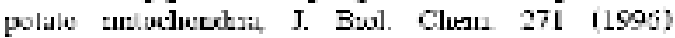
32713.2718

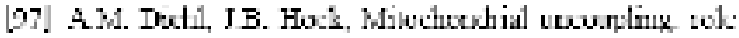
of uxcompline pomein anion carriers and relationslip m

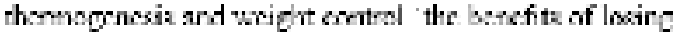

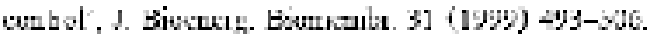

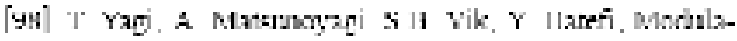
lan of Ite knuelva and lis steidy staje lets] of unterneduses of noschoudral coupled reicticca b; niusitos and vicoupless. Dischemisty 27 (19S1) 1022-_036.

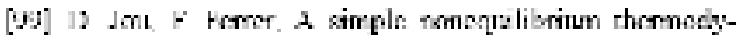

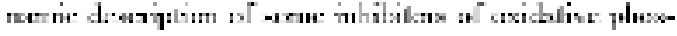
peisylatsia, J. Thuxa. Bivis. 117 (1285) 471-438.

| arrose the minchandral ineve morrlyate to stuedsed ansabolic ate. J. Tator. Dicl. 215 (1990) 267295.

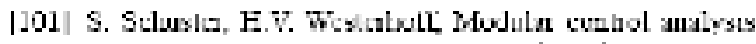
ol shypmy emzers, Busysters 19 (1959) I 15.

[102] R.E. Mutsku, S.L Sulvow, Asplisulisus of exikubad

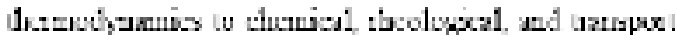

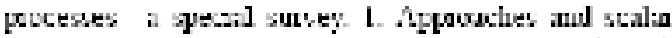
rate prosteses. J. Noc Iquilit. Thecwodya. 20 (1995) 205229

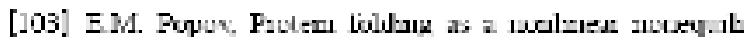
borne thennodynance pocesses, Iiocben. Mol. Dusl. lnt $17(1999) \quad 11$ ? 45 ?

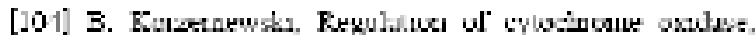

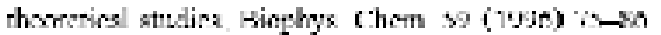

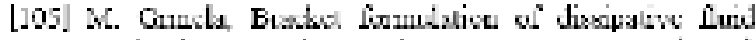

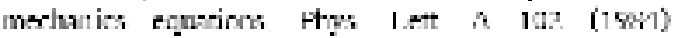
34>-3!x

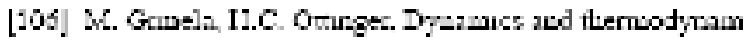
iss of complex fuidi develepment of a (it wiskic:

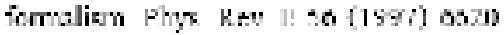

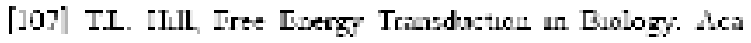
denir New Yhrk 1977

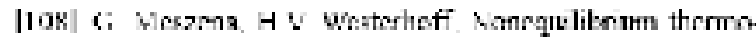

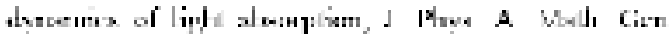
12 $\langle 1999\} ? 01 \% 1$

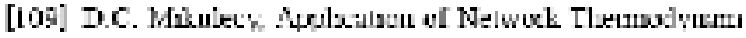

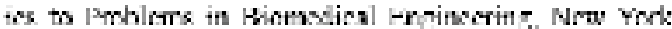

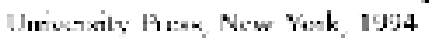

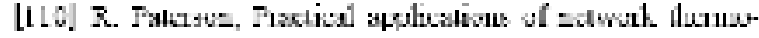

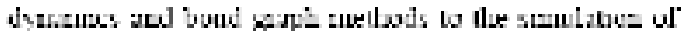

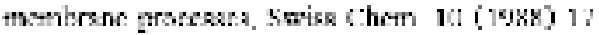

[1:1] D.C. Mikulecky; Cta porte what art bey good for.

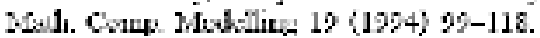

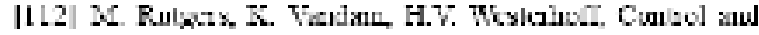

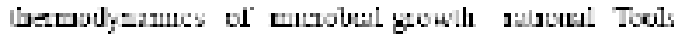
for bicengunetug. Crit. Res: Dootechnel. 11 (1991) $367-325$

|IL3| X.R. Dallz, JJ. Stzuna, J.P. Mualaya, RC natsuk

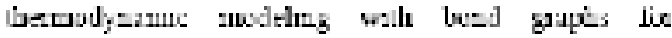

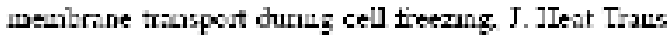
fo 110 (1988) $938-945$.

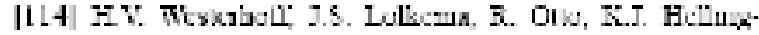

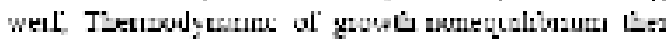
wadyuaics bectenal growth the pheconendogeal

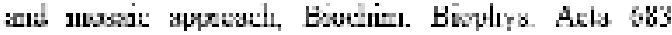
(1982) $18 .-220$

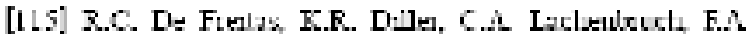

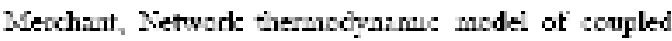

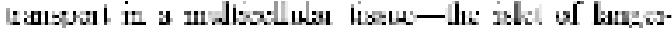

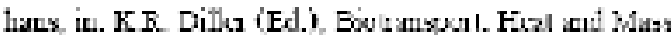

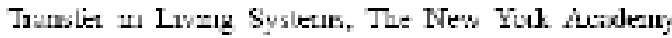
of S:ims Now rodk, $19 \%$

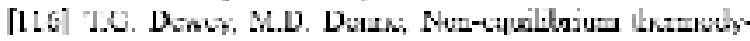
nacies of modcales coviluiva. J. Thure Bsul 193 (1958) 593559 .

[1 .7] T.G. Dewey, Ligentareve couplexty of a protein Phys. R.s. E⿱ $54\langle 1 \% 6)$ K3\%-K41

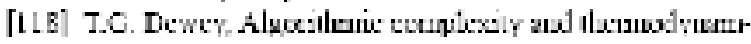

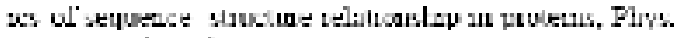
Rsv: I 56 (1957) 1515 1552.

[1:9] 7. Slbert, D Drax: J. Lewis M. Ratt K. Robers, J.D.

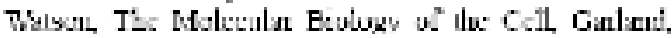
New Youk, IS91.

[120] I JuLcher, A Ajdari, J Prost, Modelung nolecular wotor, Rew Mod. Phys. 69 (1997) 126\% 1281.

[121] A Kathalsisy A Oplatse, Mertano clertiral roner

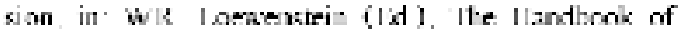
Senucry Plyyolog;, nol. 1. Pnuxuples of Receptor Phyaology. Spricge: Verlog, Deriar 1971. pg. 117.

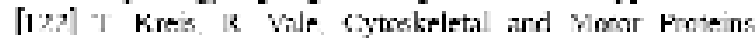
Oxfom Iniverting Prese, New Yotk. 1354

[1'3] 3 IJimrnth, Gi Kaim, 1. Natthey, The mowe of th: ATP syuhzse, Duchum. Duoghys. Aet Dusenerg 1165

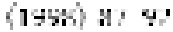

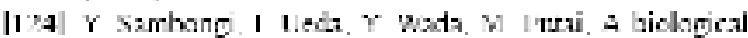
malemalar mstar, mston rmalocatinn AT' schthase ualtudveylunury apgroacl for a vaque menibras

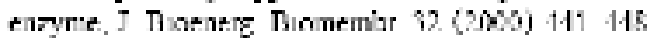

